ARTICLE

https://doi.org/10.1038/s41467-020-16051-9

\title{
SH3RF3 promotes breast cancer stem-like properties via JNK activation and PTX3 upregulation
}

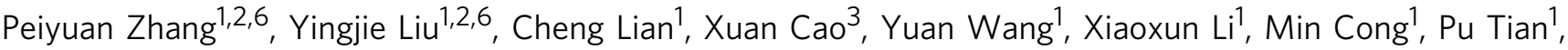 \\ Xue Zhang ${ }^{1}$, Gang Wei ${ }^{3}{ }^{3}$, Tong Liu ${ }^{4,5 凶} \&$ Guohong Hu (iD) ${ }^{1 凶}$
}

Cancer stem-like cells (CSCs) are the tumorigenic cell subpopulation and contribute to cancer recurrence and metastasis. However, the understanding of CSC regulatory mechanisms remains incomplete. By transcriptomic analysis, we identify a scaffold protein SH3RF3 (also named POSH2) that is upregulated in CSCs of breast cancer clinical tumors and cancer cell lines, and enhances the CSC properties of breast cancer cells. Mechanically, SH3RF3 interacts with the c-Jun N-terminal kinase (JNK) in a JNK-interacting protein (JIP)-dependent manner, leading to enhanced phosphorylation of JNK and activation of the JNK-JUN pathway. Further the JNK-JUN signaling expands CSC subpopulation by transcriptionally activating the expression of Pentraxin 3 (PTX3). The functional role of SH3RF3 in CSCs is validated with patient-derived organoid culture, and supported by clinical cohort analyses. In conclusion, our work elucidates the role and molecular mechanism of SH3RF3 in CSCs of breast cancer, and might provide opportunities for CSC-targeting therapy.

\footnotetext{
${ }^{1}$ Shanghai Institute of Nutrition and Health, Shanghai Jiao Tong University School of Medicine (SJTUSM) \& Chinese Academy of Sciences, Shanghai, China. ${ }^{2}$ CAS Key Laboratory of Tissue Microenvironment and Tumor, Shanghai Institute of Nutrition and Health, University of Chinese Academy of Sciences, Chinese Academy of Sciences, Shanghai, China. ${ }^{3}$ CAS Key Laboratory of Computational Biology, Collaborative Innovation Center for Genetics and Developmental Biology, CAS-MPG Partner Institute for Computational Biology, Shanghai Institute of Nutrition and Health, Chinese Academy of Sciences, Shanghai, China. ${ }^{4}$ Department of Breast Surgery, Harbin Medical University Cancer Hospital, Harbin, China. ${ }^{5}$ The Institute of Cancer Prevention and Treatment, Harbin Medical University, Harbin, China. ${ }^{6}$ These authors contributed equally: Peiyuan Zhang, Yingjie Liu. ${ }^{凶}$ email: liutong@hrbmu.edu.cn; ghhu@sibs.ac.cn
} 
C ancer represents a major threat of human health. Due to high tumor heterogeneity, the efficacy of most cancer therapies is often hindered by the existence of minor subpopulation of cancer cells with enhanced therapy resistance, which leads to tumor recurrence and eventually therapy failure. The CSC theory offers one explanation of tumor heterogeneity ${ }^{1,2}$ and states that CSCs, also referred as tumor-initiating cells, are the cell subpopulation in tumors equipped with tumor-initiating capabilities of unlimited self-renewal and differentiation ${ }^{3,4}$. More importantly, CSCs display enhanced resistance to chemotherapies and radiotherapies, and are often considered to account for poor therapeutic response, recurrence, and metastasis. The CSC theory has been well tested in breast cancer. Identified early in this century, breast cancer stem-like cells (BCSCs) were originally characterized by high expression of CD44 and low or negative expression of CD245. Later, another type of BCSCs was also discovered with high aldehyde dehydrogenase (ALDH) activity ${ }^{6}$. Although a number of studies have identified the intracellular and microenvironmental cues that regulate breast cancer stemness ${ }^{7,8}$, our understanding toward CSCs in breast cancer remains incomplete. Elucidating the molecular underpinning of BCSCs, especially the common regulators of different BCSC types with clinical relevance, will be important for rational designing of CSC-targeting strategies in cancer treatment.

SH3RF3 (SH3 domain containing ring finger 3), also known as $\mathrm{POSH} 2$ (plenty of SH3 domains protein 2), is a recently identified protein with four Src homology 3 (SH3) domains and a Ring finger domain ${ }^{9}$. SH3RF3 is found to function as a scaffold interacting with PAK2 and RAC1 through its $\mathrm{SH} 3$ domains ${ }^{9,10}$. In addition, the Ring finger domain might also confer a selfubiquitinating enzymatic activity to the protein ${ }^{10}$. Clinical analyses have shown that SH3RF3 expression is associated with acute lymphoblastic leukemia ${ }^{11}$, HIV-associated neurocognitive disorder $^{12}$, and late-onset familial Alzheimer's disease ${ }^{13}$. However, the functional role and mechanism of SH3RF3 in pathological processes, especially in cancer, are largely unexplored. In this study, we found SH3RF3 could upregulate the expression of Pentraxin 3 (PTX3) to promote stem-like traits of breast cancer cells. PTX3 is a secretory protein belonging to the pentraxin family. As a pattern recognition receptor, PTX3 plays critical roles in innate immunity ${ }^{14}$, tissue repair, and remodeling ${ }^{15}$. PTX3 dysregulation is also widely observed in cancer ${ }^{16-20}$. Many studies have established the potential of PTX3, especially its serological level, as a biomarker for a number of pathological conditions including cancer ${ }^{16,17,20,21}$. However, the roles of PTX3 in various cancers have been seemingly controversial, with reports showing both tumor-suppressing and promoting effects of PTX3 in tumor stemness, growth and metastasis ${ }^{22}$. Therefore, a clear definition of its functions in specific cancer types is of practical importance for clinical application of PTX3 in diagnosis and therapeutics.

In this study, we show that the scaffold protein SH3RF3 is highly expressed in breast cancer CSCs and reveal its function to enhance the cancer cell stemness by regulating PTX3.

\section{Results}

SH3RF3 is associated with CSC properties in breast cancer. To identify regulatory molecules in BCSCs, we sorted HMLER, a human breast cancer cell line often used in CSC studies ${ }^{23}$, into a $\mathrm{CD}_{4}{ }^{+} \mathrm{CD} 24^{-}$(termed as $\mathrm{CD} 44 \mathrm{H}$ subsequently) subpopulation and a $\mathrm{CD} 44^{-} \mathrm{CD} 24^{+}$(termed as $\mathrm{CD} 44 \mathrm{~L}$ subsequently) subpopulation via flow cytometry (Fig. 1a). The CD44L cells were further cultured in consecutive tumorsphere passages, resulting in a new subpopulation named as CD44LS. Both the $\mathrm{CD} 44 \mathrm{H}$ and CD44LS sublines displayed enhanced tumorsphere formation (Fig. 1b) and resistance to chemotherapeutic drugs including paclitaxel and doxorubicin (Fig. 1c), corroborating CSC enrichment in these two sublines. Interestingly, CD44H and CD44LS showed distinct expression pattern of CSC markers. While $\mathrm{CD} 44 \mathrm{H}$ was mainly CD $44^{+} \mathrm{CD} 24^{-}$and contained few $\mathrm{ALDH}^{+}$ cells, the CD44LS cells displayed much higher expression of ALDH (Fig. 1d). Notably, although sphere culturing of CD44L cells led to gradual increase of CD44 expression (Supplementary Fig. 1a), the CD $44^{+} \mathrm{CD} 24^{-}$content of CD44LS remains much lower than that of $\mathrm{CD} 44 \mathrm{H}$ cells (Fig. 1d). These observations were in line with the previous notion that $\mathrm{CD} 44^{+} \mathrm{CD} 24^{-}$and $\mathrm{ALDH}^{+}$ mark distinct BCSC subpopulations ${ }^{24}$. Then we analyzed the transcriptomic profiles of CD44L, CD44H, and CD44LS via RNA sequencing, and identified 588 upregulated and 706 downregulated genes in $\mathrm{CD} 44 \mathrm{H}$ and CD44LS as compared with CD44L (Supplementary Data File 1).

To ensure the clinical relevance of these CSC-associated genes, we further analyzed the transcriptomic datasets of two clinical cohorts. First we analyzed the Cancer Genome Atlas (TCGA) breast cancer cohort by single-sample gene set enrichment analysis (ssGSEA ${ }^{25}$ ), with a CD $44^{+}$CD $24^{-}$BCSC signature previously identified in human breast carcinomas ${ }^{26}$, and then the genes with expression highly correlated with the BCSC ssGSEA scores in the TCGA samples were selected. In addition, we analyzed the expression profiles of a cohort of patient-derived tumorspheres versus the corresponding primary tumors (GSE751527), to identify the genes differentially expressed in the tumorspheres. These clinical analyses resulted in 76 genes (41 upregulated and 35 downregulated) that were associated with both the $\mathrm{CD} 44^{+} \mathrm{CD} 24^{-}$signature and the tumorsphere-forming capacity in clinical tumors (Supplementary Table 1).

Finally we examined the overlap of these 76 genes with the above CSC-related genes identified in HMLER sublines, and found 21 genes (12 upregulated and 9 downregulated) in common (Fig. 1e). Many of the genes in the list, including GLI2 $2^{28}$, COL6A2 $2^{29}$, GRP $124^{30}$, SERPINF1 $1^{23,31}, M M P 2^{32}$, TIMP2 $2^{33}, G R H L 2^{34}$, and ESRP1 $1^{35}$, have been previously shown to regulate stemness of normal or cancer cells, or epithelial-mesenchymal transition (EMT), a process highly related to CSC properties ${ }^{36}$. Notably, SH3RF3, which had not been previously implicated in CSC regulation, was among the genes highly upregulated in CSC-enriched tumors and cancer cell sublines (Fig. 1e). Further real-time reverse transcription PCR (qRT-PCR) and Western blot analyses confirmed the elevated expression of SH3RF3 in $\mathrm{CD} 44 \mathrm{H}$ and CD44LS sublines of HMLER (Fig. 1f). In addition, when we isolated CD $44^{+} \mathrm{CD} 24^{-}$ and $\mathrm{CD} 44^{-} \mathrm{CD} 24^{+}$subpopulations of two additional human cell lines HMLE and MCF10AT, upregulation of SH3RF3 in the $\mathrm{CD} 44^{+} \mathrm{CD} 24^{-}$subpopulation was also observed (Fig. 1f), indicating a role of $\mathrm{SH} 3 \mathrm{RF} 3$ in BCSC regulation.

SH3RF3 promotes CSC properties of breast cancer cells. To investigate the functional role of SH3RF3 in BCSCs, SH3RF3 was overexpressed in HMLE, MCF10AT and MDA-MB-231 cells (Fig. 2a), followed by flow cytometry analyses of CSC contents in these cell lines. SH3RF3 overexpression led to $\mathrm{CD} 44^{+} \mathrm{CD} 24^{-}$cell expansion in HMLE and MCF10AT. The $\mathrm{ALDH}^{+}$subpopulation in MDA-MB-231 was also increased (Fig. 2b). In addition, SH3RF3 overexpression significantly enhanced tumorsphere formation in all three cell lines (Fig. 2c). In Py8119, a murine breast cancer cell line derived from the PyMT-driven tumors, Sh3rf3 upregulation was also observed in the $\mathrm{ALDH}^{+}$subpopulation versus the $\mathrm{ALDH}^{-}$counterpart (Supplementary Fig. 1b), and Sh3rf3 overexpression increased the capacity of tumorsphere formation by Py8119 (Supplementary Fig. 1c and d). Further, we 
a

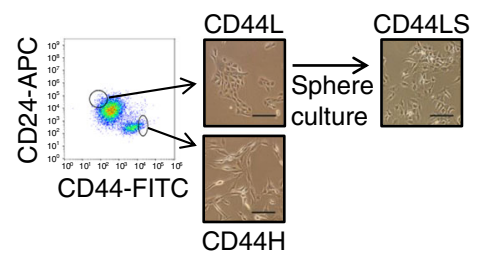

C

口CD44L 口CD44H 口CD44LS

Paclitaxel
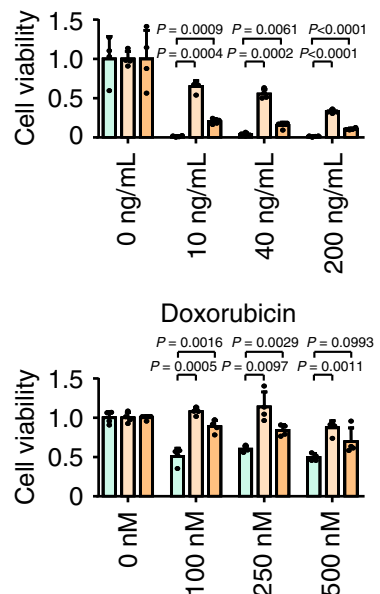

e b
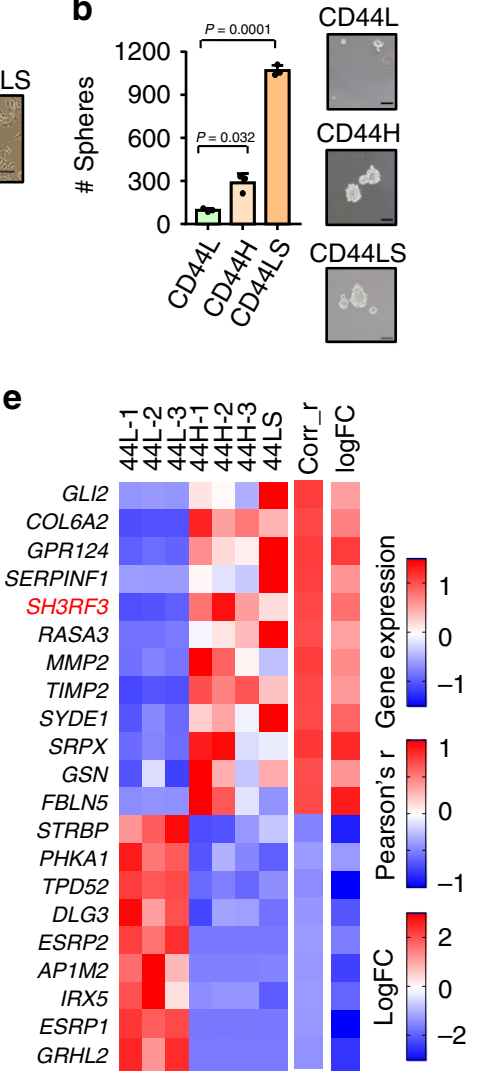

d
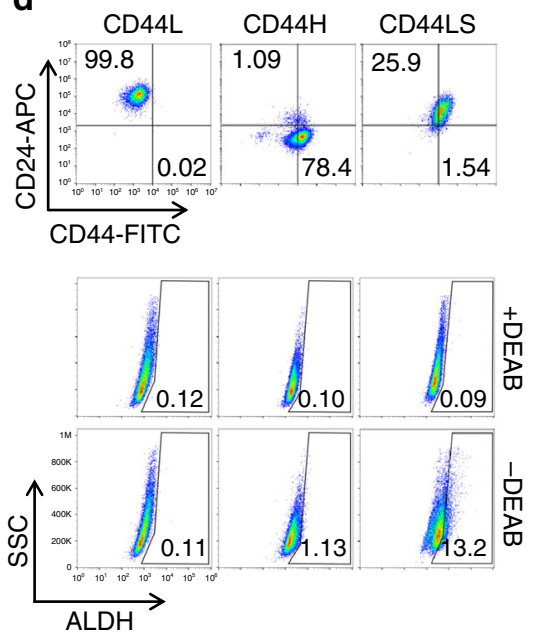

f

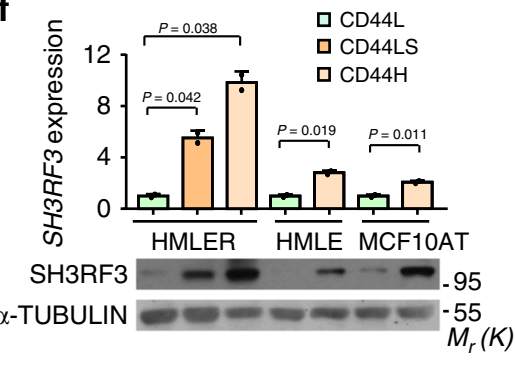

Fig. 1 SH3RF3 expression is associated with BCSC properties. a Flow chart and representative images of HMLER subline derivation. Scale bar, $100 \mu$ m. b Quantitation ( $n=3$ culturing experiments) and representative images of tumorspheres in HMLER sublines. Scale bar, $100 \mu \mathrm{m}$. c MTT assays of HMLER sublines after paclitaxel $(0-200 \mathrm{ng} / \mathrm{mL})$ and doxorubicin $(0-500 \mathrm{nM})$ treatment in various concentrations $(n=4$ treatment experiments). $\mathbf{d}$ Flow cytometry analyses of $\mathrm{CD} 44^{+} \mathrm{CD} 24^{-}$and $\mathrm{ALDH}^{+} \mathrm{CSC}$ in HMLER sublines. Numbers in the cytometry charts indicate the percentages of corresponding subpopulations. e Genes associated with CSC properties in clinical tumors and HMLER sublines. Heatmap showed their expression in HMLER sublines, Pearson's $r$ correlation to CSC signature in TCGA tumors (Corr_r) and log-fold changes of expression in tumorspheres versus primary tumors (logFC). $\mathbf{f}$ SH3RF3 expression in different sublines derived from HMLER, HMLE and MCF10AT ( $n=3$ cell culturing). Data represent mean \pm SD. Statistical significance was determined by two-tailed unpaired $t$-test $(\mathbf{b}, \mathbf{c}$, and $\mathbf{f})$. The experiments in $(\mathbf{b})$ and $(\mathbf{f})$ were repeated three times independently with similar results, and the data of one representative experiment are shown. Source data are provided as a Source data file.

analyzed the in vivo tumorigenic capacity of SH3RF3-overexpressing cancer cells via limiting dilution assays. Various numbers of MDA-MB-231 control or SH3RF3-overexpressing cells were orthotopically transplanted into female immunodeficient NSG mice. When 1000 cells were injected, all mice developed tumors and no difference was observed between the two groups. However, when fewer cells were inoculated, a stronger tumor-initiating capability was observed in the overexpression group. While only $10 \%$ of the mice injected with 40 control cells succumbed to tumors at day 28 , the tumorigenic rate was $60 \%$ for the overexpression group at the same time point, with the CSC frequency nearly tripled after $S H 3 R F 3$ overexpression (Fig. 2d). The tumor volumes were also obviously enhanced in mice inoculated with overexpressing cells (Fig. 2e). A similar phenomenon was also observed for HMLER cells in which SH3RF3 overexpression led to enhance in vivo tumor incidence in the limiting dilution assay (Supplementary Fig. 1e and f). These results suggest that $S H 3 R F 3$ overexpression could facilitate CSC properties in breast cancer cells.

To further test whether SH3RF3 is essential for CSC maintenance in breast cancer, SH3RF3 was knocked down in HMELR-CD44H and MCF10CA1h cell lines by multiple small interfering RNAs (siRNAs) or short hairpin RNAs (shRNAs) (Fig. 3a). Flow cytometry analyses showed an obvious shift to lower CD44 expression in HMELR-CD44H and a marked decrease of $\mathrm{ALDH}^{+}$fraction in MCF10CA1h after SH3RF3 knockdown (Fig. 3b). SH3RF3 knockdown also impaired the capability of both HMELR-CD44H and MCF10CA1h to form tumorspheres (Fig. 3c). More importantly, limiting dilution assays showed that $S H 3 R F 3$ knockdown diminished the tumorigenic capability of MCF10CA1h cells in mice after orthotopic transplantation (Fig. 3d, e). Altogether, these results suggested the role of SH3RF3 in CSC promotion and maintenance in breast cancer cells.

PTX3 is regulated by SH3RF3 and enhances BCSC properties. To elucidate the mechanism of SH3RF3 in BCSC regulation, we analyzed the transcriptomic profiles of MCF10AT cells by RNA sequencing to identify the genes with expression significantly changed after SH3RF3 overexpression. We further overlapped these genes with those differentially expressed in HMLER sublines. The analysis resulted in a list of 24 genes associated with both SH3RF3 expression and BCSC properties (Fig. 4a). In the list, $P T X 3$, encoding a secreted protein of the pentraxin family, was one of the top ranked genes. The regulation of PTX3 by $S H 3 R F 3$ was verified in RNA and protein levels after $S H 3 R F 3$ overexpression and knockdown (Fig. 4b). In addition, analysis of the Cancer Cell Line Encyclopedia expression database ${ }^{37}$ showed a strong positive correlation of SH3RF3 and PTX3 expression in 
a
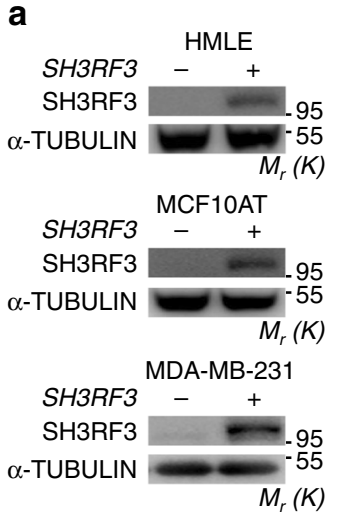

b
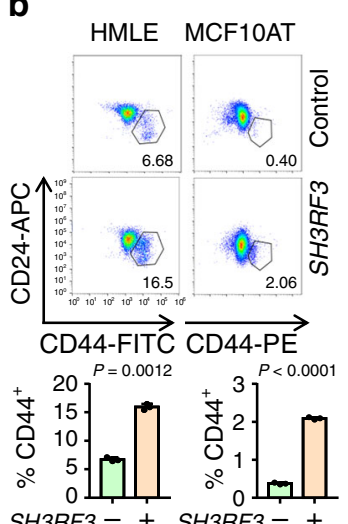
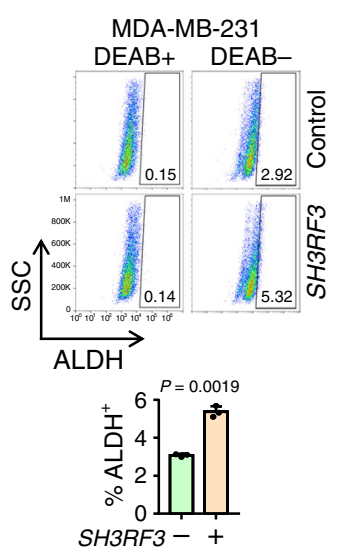

c
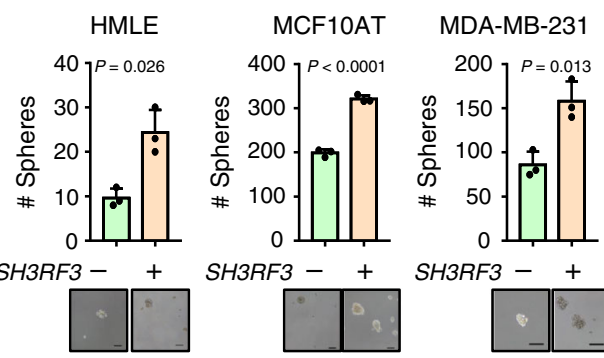

SH3RF3 - +

d

\begin{tabular}{|c|c|c|c|c|c|}
\hline $\begin{array}{c}\text { No. of cells } \\
\text { injected }\end{array}$ & 40 & 200 & 1000 & $\begin{array}{c}\text { CSC } \\
\text { frequency }\end{array}$ & $P$ value \\
\hline Control & $1 / 10$ & $4 / 10$ & $10 / 10$ & $1 / 308$ & 0.017 \\
\hline SH3RF3 & $6 / 10$ & $7 / 10$ & $10 / 10$ & $1 / 105$ & \\
\hline
\end{tabular}

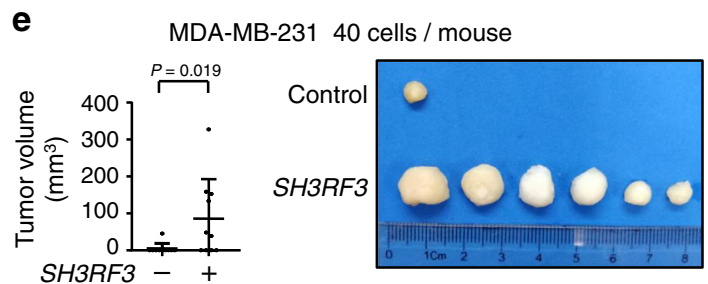

Fig. 2 SH3RF3 promotes CSC properties of breast cancer cells. a SH3RF3 overexpression in HMLE, MCF10AT and MDA-MB-231 cells. b Flow cytometry analyses of the $\mathrm{CD} 44^{+} \mathrm{CD} 24^{-}$subpopulations in HMLE and MCF10AT, and the ALDH ${ }^{+}$subpopulation in MDA-MB-231. Numbers in the flow cytometry charts indicate the CSC percentages ( $n=3$ culturing experiments). c Quantitation and representative images of tumorsphere formation in SH3RF3overexpressing breast cancer cells ( $n=3$ culturing experiments). Scale bar, $100 \mu \mathrm{m}$. $\mathbf{d}$ In vivo tumor formation of the mice injected with serial dilutions of MDA-MB-231 cells at day 28. e Tumor images and volumes $(n=10$ mice) in mice injected with 40 control and SH3RF3-overexpressing MDA-MB-231 cells. Data represent mean \pm SD. Statistical significance was determined by two-tailed unpaired $t$-test ( $\mathbf{b}$ and $\mathbf{c}$ ), chi-squared test (d) or Mann-Whitney $U$-test (e). The experiments in (a), (b) (upper), and (c) were repeated three times independently with similar results, and the data of one representative experiment are shown. Source data are provided as a Source data file.

breast cancer cell lines (Fig. 4c), further confirming that PTX3 is a downstream gene regulated by SH3RF3.

Next, the functional role of PTX3 in BCSCs was studied. PTX3 overexpression in HMLE, HMLER and MCF10AT (Fig. 4d and Supplementary Fig. 2a) led to obvious enhancement of tumorsphere formation by these cell lines (Fig. 4e and Supplementary Fig. 2b). PTX3 overexpression resulted in increases of CD44 CD24- subpopulation of HMLE (Fig. 4f, g) and in vivo tumorigenesis by HMLER (Fig. 4h). Further, the transcriptomes of control and PTX3-overexpressing HMLER cells were profiled by RNA sequencing, followed by gene set enrichment analysis (GSEA) of the transcriptomic profiles with a number of previously identified CSC and mammary stem cell (MaSC)related gene sets ${ }^{26,38,39}$. The GSEA analysis showed that the gene sets upregulated in CSCs or MaSCs (IGS_UP, MET_CD44_UP, N_CD44_UP) were significantly enriched in the PTX3-overexpressing cells, while the gene sets downregulated in CSCs or MaSCs (MET_CD44_DOWN, MaSC_DOWN) were enriched in the control cells (Fig. 4i and Supplementary Fig. 2c), corroborating a role of PTX3 in CSC regulation.

Interestingly, GSEA analysis also revealed the upregulation of Hedgehog and Yes associated protein (YAP) signaling pathways after PTX3 overexpression. The gene sets upregulated by Sonic Hedgehog (SHH_UP) ${ }^{40}$ or its downstream transcription factor GLI1 (GLI1_UP) ${ }^{4}$, and by YAP (YAP_UP) ${ }^{42}$ were significantly enriched in PTX3-overexpressing cells as compared with control cells (Fig. 4j and Supplementary Fig. 2d). Upregulation of Hedgehog and YAP downstream genes after PTX3 overexpression was also confirmed by qRT-PCR (Fig. 4k). Previously many studies have showed that activated Hedgehog ${ }^{43}$ and $\mathrm{YAP}^{44}$, which is the transcription factor effector of Hippo pathway, play essential roles in the regulation of cancer stemness. Thus, our data support a functional role of PTX3 in BCSCs, likely by regulating the Hedgehog and Hippo-YAP pathways.

PTX3 mediates the role of SH3RF3 in BCSC regulation. Based on the above observations that SH3RF3 enhances the expression of PTX 3 and that PTX3 plays a role in BCSC regulation, we hypothesized that $P T X 3$ is the main downstream gene of SH3RF3 to mediate its role in BCSCs. To test this hypothesis, we first analyzed whether the transcriptomic changes caused by SH3RF3 overexpression and PTX3 overexpression were similar. The ssGSEA analysis showed that the genes with differential expression by SH3RF3 and PTX3 overexpression were coordinately expressed in breast cancer cell lines (Fig. 5a). In addition, the genes sets enriched in PTX3-overexpressing cells were also enriched after SH3RF3 overexpression (Fig. 5b). Furthermore, the Hedgehog and Hippo-YAP downstream genes that were regulated by PTX3 were also regulated by SH3RF3 overexpression (Fig. 5c). These data indicated that SH3RF3 and PTX3 result in consistent transcriptomic effects of cancer cells, especially on the genes related to cancer stemness.

Next we tested whether PTX3 inhibition is able to rescue the effect of SH3RF3 on CSCs by siRNA silencing of PTX3 in control and SH3RF3-overexpressing MDA-MB-231 cells (Fig. 5d and Supplementary Fig. 3a). Flow cytometry analyses revealed that SH3RF3 expanded the $\mathrm{ALDH}^{+}$CSC subpopulation in MDA-MB231, while PTX3 inhibition shrank the CSC population and effectively blocked the effect of $S H 3 R F 3$ overexpression (Fig. 5e, f and Supplementary Fig. 3b). Furthermore, PTX3 silencing impaired the promoting effect of SH3RF3 on tumorsphere formation in both MDA-MB-231 and HMLE cells (Fig. 5g and Supplementary Fig. 3c). In addition, shRNA knockdown of PTX3 in SH3RF3-overexpressing MDA-MB-231 cells restrained the 
a

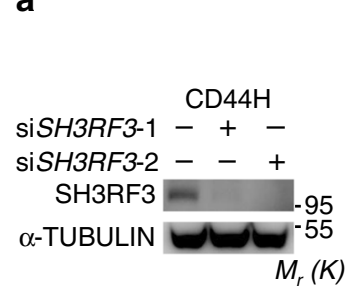

b

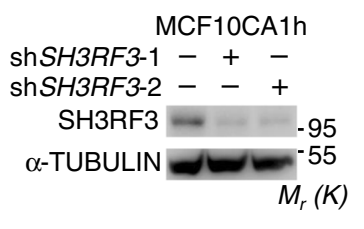

d

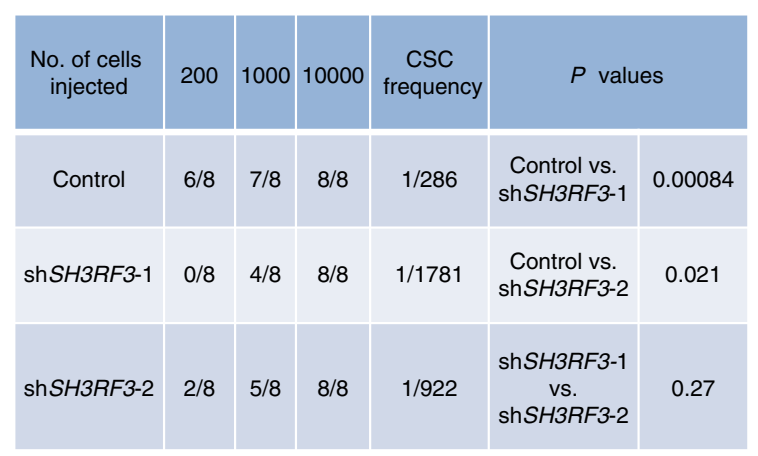

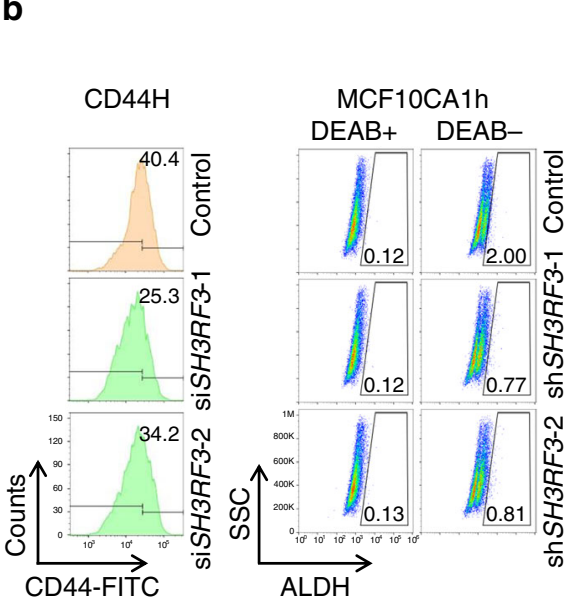

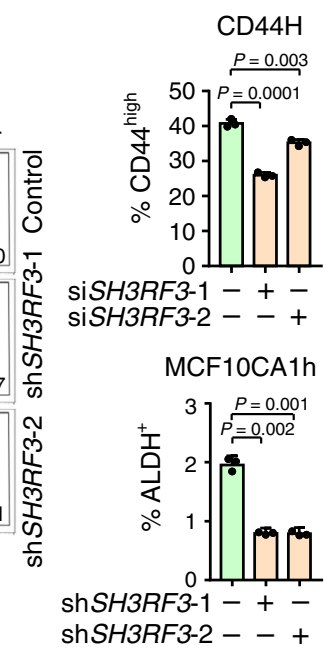

C

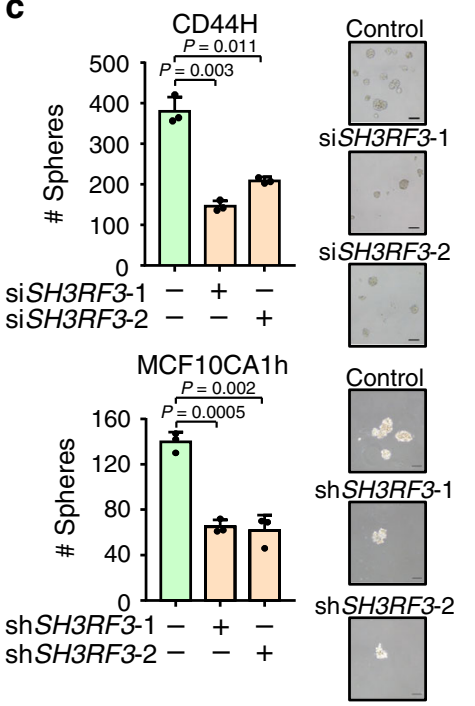

e

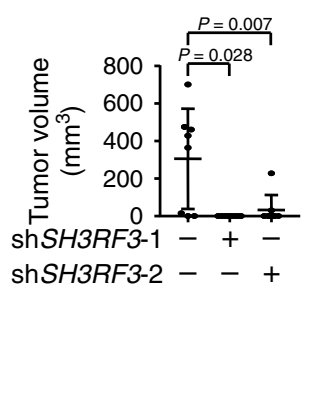

MCF10CA1h 200 cells / mouse

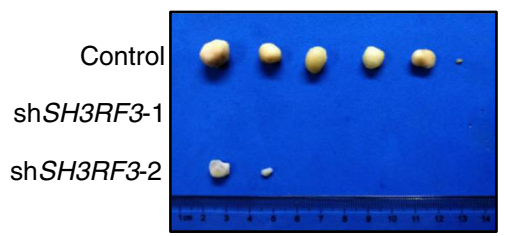

Fig. 3 SH3RF3 knockdown impairs CSC traits of breast cancer cells. a SH3RF3 knockdown in HMLER-CD44H and MCF10CA1h cells. b Flow cytometry analyses of the CD44high subpopulation in HMLER-CD44H and the $\mathrm{ALDH}^{+}$subpopulation in MCF10CA1h ( $n=3$ cell culture experiments). c Quantitation and representative images of tumorsphere formation of breast cancer cells with SH3RF3 knockdown ( $n=3$ sphere culture experiments). Scale bar, $100 \mu$ m. d In vivo tumor formation of the mice injected with serial dilutions of MCF10CA1h at day 42. e Tumor images and volumes ( $n=8$ mice) in mice injected with 200 control or SH3RF3 knockdown MCF10CA1h cells. Data represent mean \pm SD. Statistical significance was determined by two-tailed unpaired $t$-test (b and $\mathbf{c}$ ), chi-squared test (d) or Mann-Whitney U-test (e). The experiments in (a), (b) (left), and (c) were repeated three times independently with similar results, and the data of one representative experiment are shown. Source data are provided as a Source data file.

enhancement of in vivo tumorigenesis by $S H 3 R F 3$ (Fig. 5h). These results collectively indicated that PTX3 acts at the downstream of SH3RF3 to promote CSC features of breast cancer cells.

SH3RF3 enhances PTX3 expression through JNK-JUN pathway. To investigate how SH3RF3 enhances PTX3 expression, we tested whether several CSC-related signaling pathways, including $\mathrm{PI} 3 \mathrm{~K}, \mathrm{JNK}, \mathrm{WNT}$, and NF- $\kappa \mathrm{B}$, were involved in SH3RF3 regulation of PTX3 expression. First, HMLE cells were treated with a PI3K inhibitor BKM120. It was found that BKM120 inhibited PTX3 expression and tumorsphere formation (Supplementary Fig. $4 \mathrm{a}$ and $\mathrm{b}$ ), consistent to the previous report showing that PTX3 was a target gene regulated by PI3K-AKT signaling ${ }^{45}$. However, SH3RF3 overexpression was still able to upregulate PTX3 expression (Supplementary Fig. 4a) and tumorsphere formation (Supplementary Fig. 4b) in the presence of PI3K inhibition. Importantly, SH3RF3 caused no difference in AKT phosphorylation (Supplementary Fig. 4c), further suggesting that SH3RF3 regulates PTX3 in a PI3K-independent pathway. In addition, we found that the JNK inhibitor (SP600125), but not the WNT or NF- $\mathrm{BB}$ inhibitors (XAV939 and BAY11-7082), could dramatically suppress the expression level of PTX3 and eliminate the effect of SH3RF3 in HMLE cells (Fig. 6a). In addition, only
SP600125 was able to completely block the promotion of tumorsphere formation by SH3RF3, although the other inhibitors also partially reduced tumorspheres (Fig. 6b). The rescue effect of SP600125 on SH3RF3-regulated PTX3 expression and tumorsphere formation was also observed in MDA-MB-231 cells (Fig. 6c, d).

Thus we further investigated the effect of SH3RF3 on the JNK signaling pathway, and found that SH3RF3 overexpression led to increased phosphorylation of JNK1, JNK2, and the downstream transcription factor JUN, while SH3RF3 knockdown inhibited JNK and JUN phosphorylation in multiple cell lines (Fig. 6e and Supplementary Fig. 4d). In addition, a list of known JUN-target genes, including MMP3, CD44, EGFR, ENPP2 ${ }^{46}$, and MGST1 ${ }^{47}$, were markedly upregulated in cancer cells with SH3RF3 overexpression (Fig. 6f). These results suggested that SH3RF3 regulates $P T X 3$ expression by activating the JNK pathway to promote BCSC properties.

Further, we studied the mechanism of PTX3 regulation by JNK signaling. JUN is the transcription factor downstream of JNK and activates target gene expression by forming the AP-1 heterodimer complex. Chromatin-immunoprecipitation (ChIP)-qPCR assays revealed the binding of JUN on PTX3 promoter (Fig. $6 \mathrm{~g}$ and Supplementary Fig. 4e). In addition, JUN could directly activate the transcriptional activity of PTX3 promoter, as shown by 
a

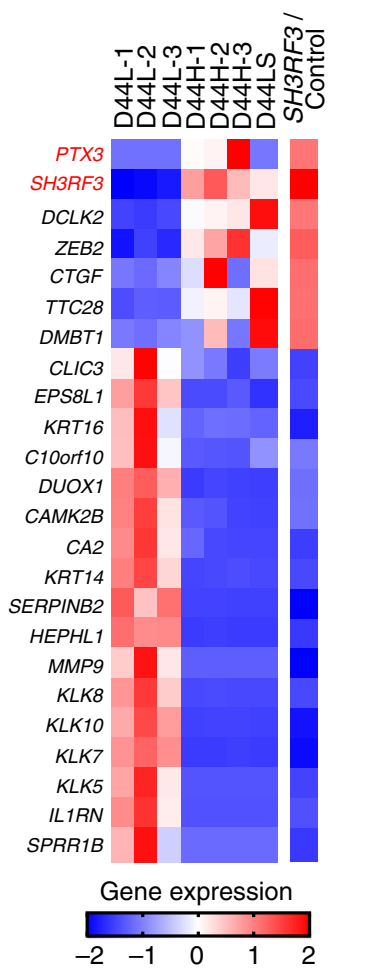

Fold Change (SH3RF3/ Control)

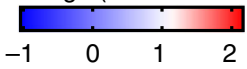

b
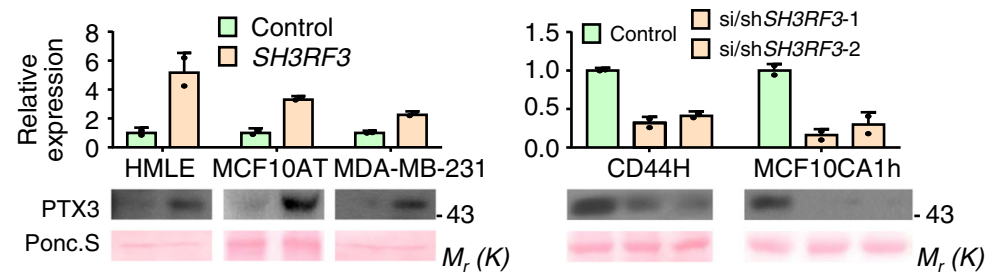

d

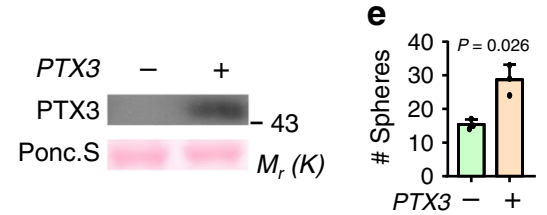

h

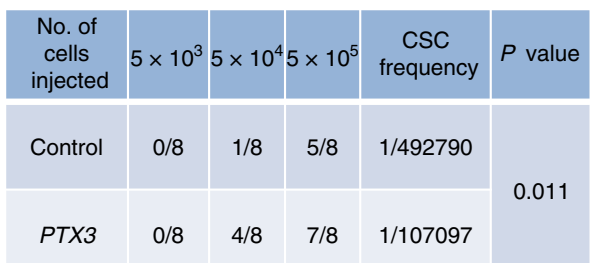

j

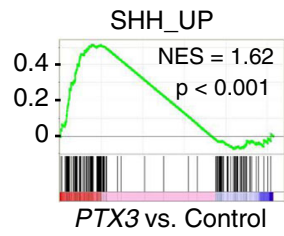

f

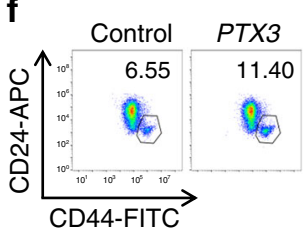

i
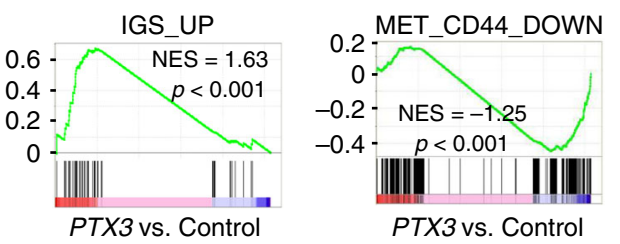

k

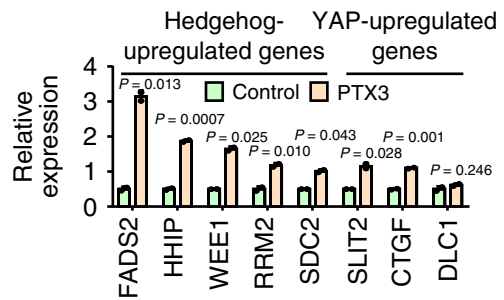

Fig. 4 PTX3 is regulated by SH3RF3 and enhance BCSC properties. a Expression heatmap of the genes differentially expressed in HMLER sublines and regulated by SH3RF3 overexpression. b PTX3 mRNA expression and its extracellular protein levels in conditioned media of various cancer cell lines after SH3RF3 overexpression and knockdown ( $n=3$ cell culturing). c Correlation of SH3RF3 and PTX3 mRNA levels in breast cancer cell lines ( $n=51$ cell lines). d Extracellular PTX3 levels in conditioned media of PTX3-overexpressing and control HMLE cells. e Tumorsphere formation of HMLE after PTX3 overexpression ( $n=3$ culturing experiments). $\mathbf{f}, \mathbf{g}$ Flow cytometry analysis (f) and quantitation ( $\mathbf{g}$ ) of CD44+CD24- CSC subpopulation in PTX3overexpressing and control HMLE cells. Scale bar, $100 \mu \mathrm{m} ; n=3$ cell culturing experiments. $\mathbf{h}$ Tumor incidence in the mice injected with various numbers of HMLER cells at day 90. i GSEA analyses of CSC and MaSC-related gene sets in PTX3-overexpressing versus control cells. $\mathbf{j}$ GSEA analyses of Hedgehog and Hippo-regulated gene sets in PTX3-overexpressing versus control cells. k mRNA levels of Hedgehog and Hippo-regulated genes after PTX3 overexpression ( $n=3$ cell culturing experiments). Data represent mean \pm SD. Statistical significance was determined by paired $t$-test (c) or two-tailed unpaired $t$-test $(\mathbf{e}, \mathbf{g}$, and $\mathbf{k})$. The experiments in $(\mathbf{b}),(\mathbf{d}),(\mathbf{e}),(\mathbf{f})$, and $(\mathbf{k})$ were repeated three times independently with similar results, and the data of one representative experiment are shown. Source data are provided as a Source data file.

luciferase reporter assays (Fig. 6h). Analyses with serial truncations of PTX3 promoter showed that JUN mainly targeted the -206 to +52 region in the promoter (Fig. $6 \mathrm{~h}$ ). In this region three AP-1 binding sites were identified (Supplementary Fig. 4e). Mutating one or two of these binding sites partially reduced the promoter activation by JUN, while mutating all three sites completely eliminated the response to JUN (Fig. 6h). Thus, all the three sites contributed to the regulation of PTX3 expression by JUN. Collectively, these data suggested that JUN activated by SH3RF3 binds to the promoter of PTX3 to enhance its expression.

SH3RF3 contains multiple SH3 domains which mediate protein-protein interactions ${ }^{9}$. Therefore, SH3RF3 might regulate JNK phosphorylation by interacting with JNK and its kinases. Indeed, reciprocal co-immunoprecipitation (co-IP) assays showed that SH3RF3 interacted with JNK1 and JNK2 (Fig. 7a and Supplementary Fig. 5a). The binding of SH3RF3 with MAP kinase kinase 4 (MKK4) and 7 (MKK7), the two main JNK kinases, was also observed (Fig. 6b and Supplementary Fig. 5b).
The phosphorylation of JNK is coordinated in a complex of JNK and MKKs, with the JNK-interacting protein (JIPs) acting as the essential scaffold for the complex organization ${ }^{48}$. Thus we studied whether the interaction between SH3RF3 and JNK in breast cancer cells is dependent on JIPs. We found that SH3RF3 could interact with all three JIP family members JIP1, JIP2, and JIP3 in 293 T (Fig. 7c and Supplementary Fig. 5c); however, JIP3 was the only JIP gene expressed in breast cancer cells MCF10AT (Supplementary Fig. 5d). In addition, sequential IP analysis proved the presence of SH3RF3, MKK7, and JIP3 in one complex (Fig. 7d). Interestingly, SH3RF3 enhanced the interaction of JIP3 to MKK7, but had no effect on JIP3-JNK2 interaction (Fig. 7e and Supplementary Fig. 5e). Instead, JIP3 knockdown by two independent siRNAs (Supplementary Fig. 5f) obviously impaired the binding of SH3RF3 to JNK proteins (Fig. 7f) and SH3RF3promoted JUN phosphorylation in MCF10AT cells (Fig. 7g). We further analyzed SH3RF3 protein region to interact with JNK signaling complex components and found that it was the fourth 

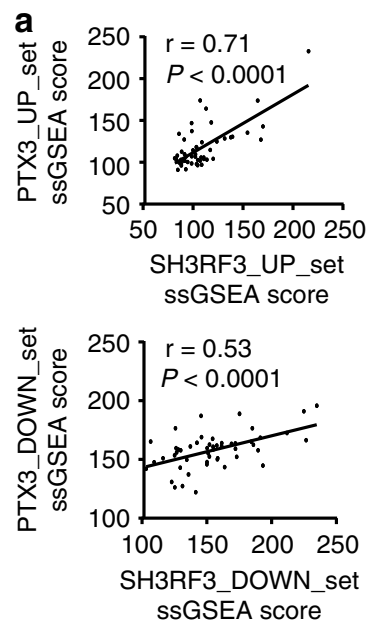

f

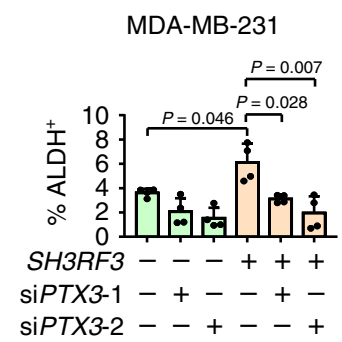

b

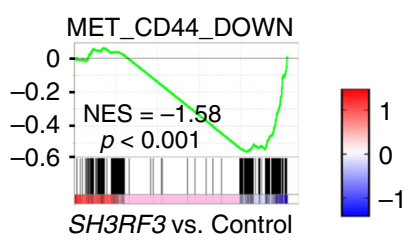

c

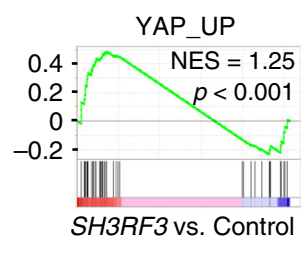

g

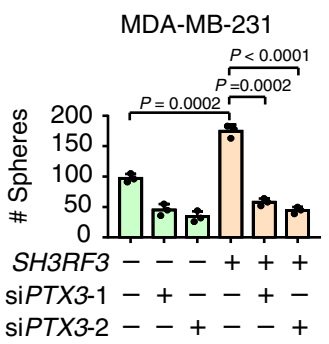

d

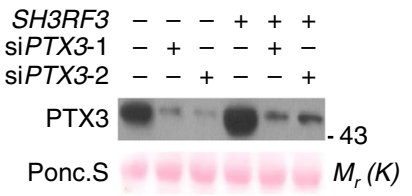

e

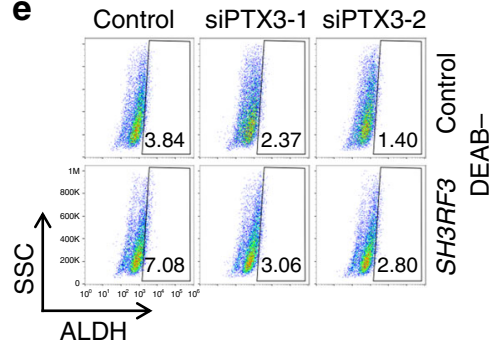

$\mathbf{h}$

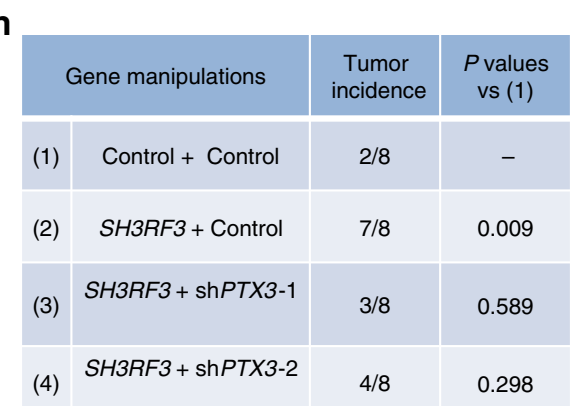

Fig. 5 PTX3 mediates the function of SH3RF3 in BCSC regulation. a Correlation of SSGSEA scores of the gene sets regulated after PTX3 and SH3RF3 overexpression in breast cancer cell lines ( $n=51$ cell lines). b GSEA analyses of CD44 or YAP-related gene sets in PTX3-overexpressing versus control cells. c Expression heatmap of Hedgehog and Hippo-regulated genes after PTX3 and SH3RF3 overexpression. d PTX3 knockdown in MDA-MB-231 with SH3RF3 overexpression. e Flow cytometry analyses of $\mathrm{ALDH}^{+}$subpopulation in MDA-MB-231 with SH3RF3 overexpression and PTX3 knockdown.

f Quantitation ( $n=4$ FACS experiments) of ALDH + CSC subpopulations in MDA-MB-231 with SH3RF3 overexpression and PTX3 knockdown.

$\mathbf{g}$ Tumorsphere quantitation in MDA-MB-231 with SH3RF3 overexpression and PTX3 knockdown ( $n=3$ culturing experiments). $\mathbf{h}$ Tumor initation in the mice injected with 40 cells of MDA-MB-231 cells at day 28. Data represent mean $\pm S D, n=3$. Statistical significance was determined by paired $t$-test (a) or two-tailed unpaired $t$-test ( $\mathbf{f}$ and $\mathbf{g}$ ). The experiments in (d), (e), and $(\mathbf{g})$ were repeated three times independently with similar results, and the data of one representative experiment are shown. Source data are provided as a Source data file.

SH3 domain that interacts with MKK7, while the first and second SH3 domains interact with JIP3 and JNK1 (Fig. 7h and Supplementary Fig. 5g). These results suggested that SH3RF3 facilitates the assembly of MKK-JNK complex in a JIP-dependent manner and promotes JNK-JUN activation.

SH3RF3 correlates with CSC properties in clinical tumors. To assess the clinical significance of SH3RF3 in CSC regulation, we analyzed the functional role of SH3RF3 in patient-derived organoids, a 3D-culture CSC assay with high clinical relevance ${ }^{49}$. Patient-derived tumor cells of three breast cancers were infected with control or SH3RF3-expressing retroviruses and seeded in matrigel with organoid culture media. Notably, SH3RF3 overexpression resulted in formation of more organoids in all three tumors, and larger sizes of the organoids (Fig. 8a, b). Similar results were observed in the organoid culture of four human gastric cancers (Fig. 8c), corroborating a role of SH3RF3 to promote CSC features of clinical tumor cells.

We then analyzed the RNA-sequencing data of the TCGA breast cancer clinical cohort. In these tumors, the expression of $S H 3 R F 3$ positively correlated to $P T X 3$, as well as the CSC markers CD44 and $A L D H 1 A 1 / 2 / 3$ (Fig. 8d). Significant correlation of PTX3 to CD44 and ALDH1A1/2/3 was also observed (Fig. 8d). Moreover, ssGSEA analyses showed that the expression of
SH3RF3 was positively correlated with the enrichment of CSC and MaSC-related gene sets in the TCGA cohort (Fig. 8e).

Next, we assessed the expression of SH3RF3 and CD44 at the protein level in a Qilu cohort of 40 breast invasive ductal carcinomas by immunofluorescence (IF) staining. The samples were divided into three groups according to the SH3RF3 IF intensities, and significantly more $\mathrm{CD} 44^{+}$cells were found in tumors with higher SH3RF3 expression (Fig. 8f, g). Meanwhile, a positive correlation between SH3RF3 signal intensities and CD44 signal intensities was also noticed in these samples (Fig. 8h). Finally, we observed a link of SH3RF3 expression to increased risk of distant metastasis (Supplementary Fig. 6a) and lower survival (Fig. 8i) of breast cancer patients in the Kaplan-Meier Plotter database ${ }^{50}$. Higher PTX3 protein expression was also associated with disease recurrence in the CPTAC breast cancer cohort (Supplementary Fig. 6b). In summary, our data confirmed that SH3RF3 plays a positive role in BCSC maintenance and is clinically associated with CSC enrichment and poor prognosis of breast cancer.

\section{Discussion}

SH3RF3 belongs to a protein family characterized with the presence of multiple SH3 domains and a Ring finger domain. In addition to SH3RF3, there are two other members, SH3RF1 


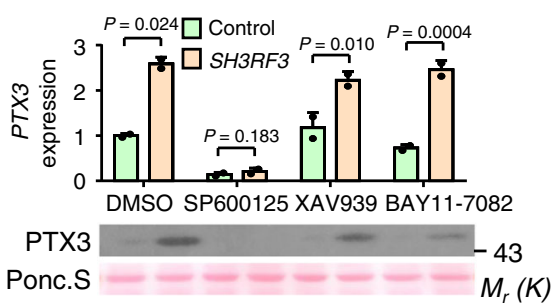

C

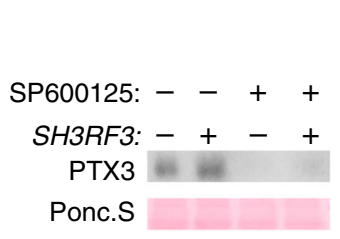

d

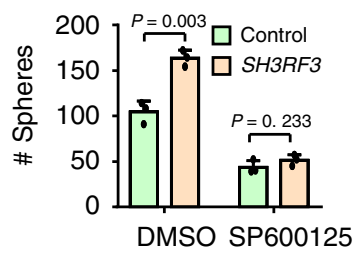

b

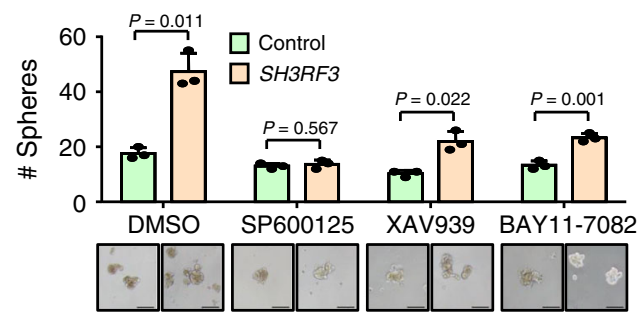

e

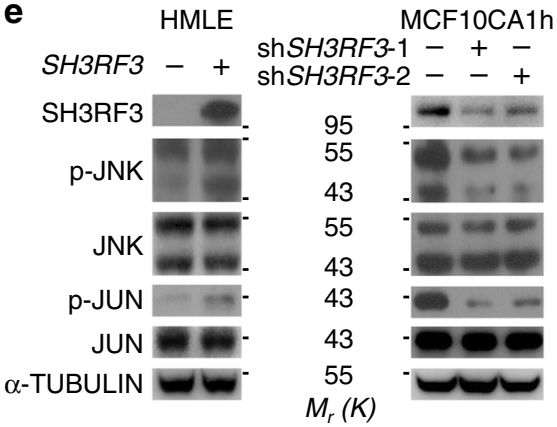

f

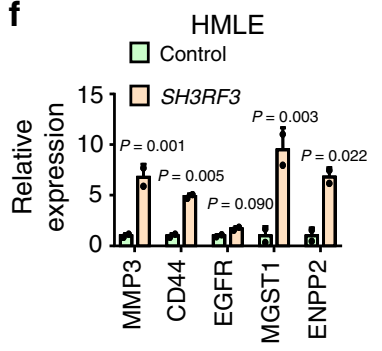

g

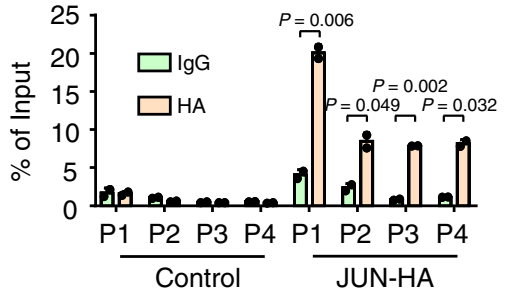

h

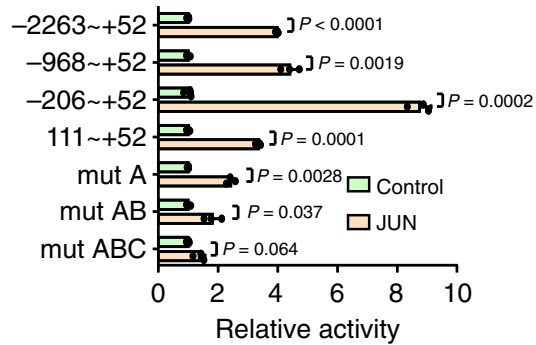

Fig. 6 SH3RF3 enhances PTX3 expression through JNK-JUN pathway. a, b PTX3 expression (a) and tumorsphere formation (b, $n=3$ culturing experiments) of SH3RF3-overexpressing and control HMLE cells treated with inhibitors of JNK (SP600125, $5 \mathrm{nM}$ ), WNT (XAV939, $5 \mathrm{nM})$, and NF-KB (BAY11-7082, $5 \mathrm{nM}$ ). c, d PTX3 expression (c) and tumorsphere formation (d, $n=3$ culturing experiments) of SH3RF3-overexpressing and control MDAMB-231 cells treated with the JNK inhibitor. e JNK and JUN phosphorylation after SH3RF3 overexpression and knockdown. $\mathbf{f}$ Expression of JUN-target genes after SH3RF3 overexpression in HMLE ( $n=3$ qRT-PCR experiments). $\mathbf{g}$ ChIP-qPCR analysis of JUN binding to PTX3 promoter in HeLa ( $n=3$ ChIP experiments). $\mathbf{h}$ Luciferase reporter analysis of PTX3 promoter in JUN-overexpressing and control HeLa cells $(n=3$ reporter assays). Data represent mean \pm SD. Statistical significance was determined by two-tailed unpaired t-test $(\mathbf{a}, \mathbf{b}, \mathbf{d}, \mathbf{f}, \mathbf{g}$, and $\mathbf{h})$. The experiments in (a-f) and $(\mathbf{g})$ were repeated three times independently with similar results, and the data of one representative experiment are shown. Source data are provided as a Source data file.

(POSH) and SH3RF2 (POSHER), in the family. Among these proteins, SH3RF1 has been relatively well studied, with previous reports showing its roles to promote cell apoptosis and regulate various pathological processes including HIV infection and cerebral ischemia ${ }^{51-53}$. SH3RF2 has been reported to inhibit cell apoptosis by antagonizing SH3RF $1^{54}$. However, the roles of these POSH members in cancer are unexplored, although dysregulation of SH3RF1 and SH3RF3 has been implicated in lung cancer and leukemia ${ }^{11,55}$. Here we report a previously unidentified role of SH3RF3 in stemness regulation of breast cancer. By transcriptomic screening of clinical tumors and cancer cell lines, we found that the expression of SH3RF3 was consistently correlated with CSC properties of breast cancer cells. More importantly SH3RF3 not only promotes, but also is required to maintain the stem-like features of cancer cells. We also demonstrate that SH3RF3 contributes to the assembly of MKK-JIP-JNK complex, leading to activation of JNK and upregulation of $P T X 3$ expression for CSC regulation in cancer cells (Fig. 8j). The effect of SH3RF3 on the JNK pathway is similar to SH3RF1, which is also a JNK activator $^{53}$, but opposite to the JNK-suppressing SH3RF2 ${ }^{54}$. This is in parallel to the structural homology of these proteins in that SH3RF2 only has three SH3 domains and our observation that the fourth SH3 domain mediates the interaction of SH3RF3 to the
JNK kinase MKK. Although the detailed mechanism of SH3RF3 to mediate the interaction of JNK and MKK is yet to be delineated, our study will expand our understanding of the POSH family proteins and CSC regulation in cancer.

In our analysis of HMLER cells, consecutive tumorsphere culturing of the non-CSC CD44L subpopulation resulted in a new subline with CSC traits including enhanced tumorsphere formation and chemoresistance. This spontaneous conversion of neoplastic non-stem cells to CSCs is concordant to previous reports $^{56}$ and highlights the plasticity of cancer cells, which is a key reason for the ultimate failure of many of current cancer therapeutics. Notably, previous studies showed that the spontaneous acquisition of CSC features of cancer cells was usually accompanied with the EMT process. In our study, although the initial CD44L and CD44H subpopulations of HMLER indeed displayed the epithelial and mesenchymal-like morphology, respectively, the CD44LS cells maintained an epithelial morphology (Fig. 1a), indicating EMT-independent mechanisms of inter-conversion between non-CSCs and CSCs.

BCSCs were also characterized by heterogeneity. A previous study demonstrated that an epithelial-like subpopulation and a mesenchymal-like subpopulation, labeled by $\mathrm{ALDH}^{+}$and $\mathrm{CD} 44$ ${ }^{+} \mathrm{CD}_{24}{ }^{-}$, respectively, exist in $\mathrm{BCSCs}^{24}$. In our study, although 
a

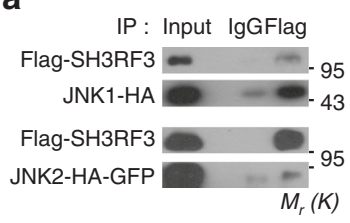

d

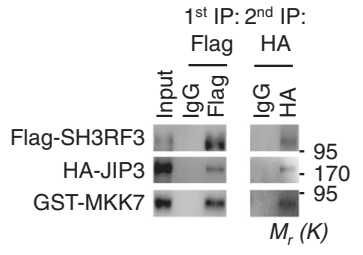

$\mathbf{f}$

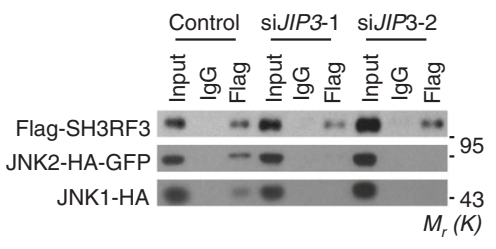

b

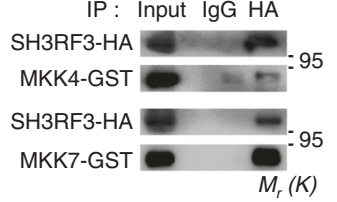

e

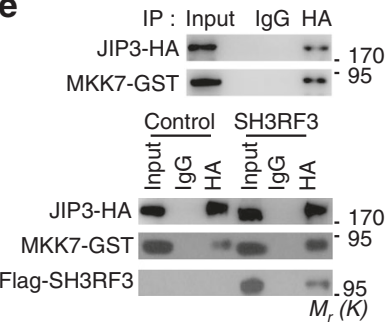

g

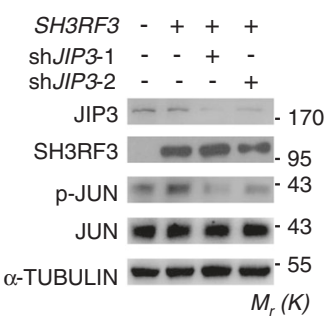

C

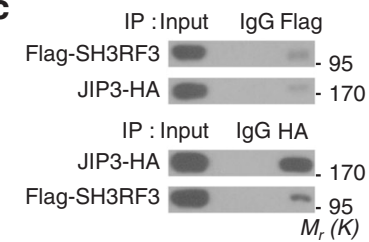

h

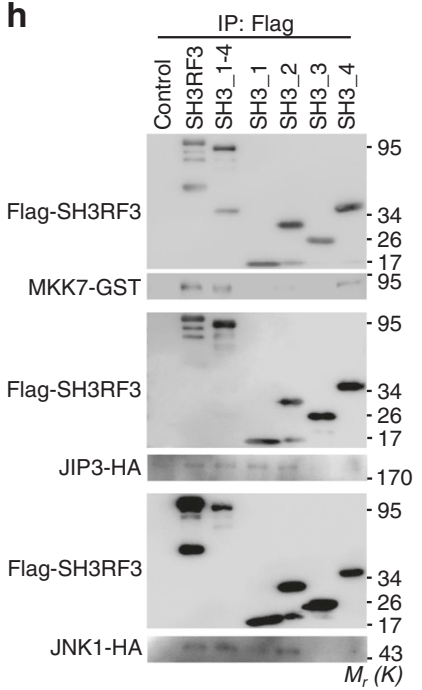

Fig. 7 SH3RF3 activates JUN by interacting with MKK and JNK. a Co-IP analyses of SH3RF3-JNK1/2 interaction in 293T cells. b Co-IP analyses of SH3RF3-MKK4/7 interaction in 293T cells. c Co-IP analyses of SH3RF3-JIP3 interaction in 293T cells. d Sequential Co-IP analyses of SH3RF3-JIP3-MKK7 interaction in 293T cells. e Co-IP analyses of JIP3-MKK7 interaction in MCF10AT (top), and with or without SH3RF3 overexpression (bottom). f Co-IP analyses of SH3RF3-JNK1/2 interaction in MCF10AT with or without JIP3 knockdown. $\mathbf{g}$ JUN phosphorylation after SH3RF3 overexpression and JIP3 knockdown in MCF10AT. h Co-IP analyses of the interactions of various SH3RF3 truncations to MKK7, JIP3, and JNK1. The experiments were repeated three times independently with similar results, and the data of one representative experiment are shown. Source data are provided as a Source data file.

both the CD44H and CD44LS sublines were characterized by enhanced stemness, $\mathrm{CD} 44 \mathrm{H}$ was mainly $\mathrm{CD} 44^{+} \mathrm{CD} 24^{-}$and only contained a minor fraction of $\mathrm{ALDH}^{+}$cells. In contrast, there was an obvious shift of the CD44LS population to higher ALDH expression (Fig. 1c). This observation corroborates the notion of CSC heterogeneity in breast cancer. More importantly, SH3RF3 can promote the expansion of both subtypes of BCSCs in different cell lines (Figs. 2b and 3b). Although different subtypes of BCSCs share little overlap and display distinct molecular profiles $^{24}$, SH3RF3 and JNK signaling might represent a common regulatory mechanism of heterogeneous BCSCs. Therefore, these results might have important implications for CSC-targeting designing in breast cancer therapeutics.

The JNK pathway is known to be involved in inflammation, apoptosis, cell differentiation, and proliferation. Recently, multiple studies have reported a critical role of JNK in CSC regulation $^{57}$. Furthermore, JNK impairment led to inhibition of the stem properties of cancer cells in colorectal cancer and breast cancer ${ }^{57-59}$. However, the mechanism of JNK activation in CSCs is still largely unknown. JNK phosphorylation and activation by MKK is dependent on the formation of the JNK signaling complex that is composed of JNK, MKK and the scaffold JIPs. Here we showed that in breast cancer cells SH3RF3 also plays a role in the complex and contributes to JNK phosphorylation. This discovery will enrich our understanding on JNK signaling, and it warrants further investigation whether SH3RF3 is a genuine component of JNK signaling complex or its role in JNK and CSC regulation is context-specific in cancer cells.

Previous reports have firmly established the role of PTX3 to promote dedifferentiation ${ }^{60}$, chemotherapy resistance ${ }^{61}$, and stem properties of basal-like breast cancer cells ${ }^{45}$. However, there are also studies reporting the silence of $P T X 3$ expression in tumors as compared with normal tissues ${ }^{18,19}$ and its onco-suppressing effects in multiple cancer types ${ }^{18,62,63}$. These seemingly contradictory observations could be reconciled by the fact that PTX3 was highly expressed only in CSC subpopulation of breast cancer and was nearly undetectable in non-CSCs (Fig. 4a and Supplementary Data File 1). Given that CSCs typically compose only a minor fraction of tumor cells, upregulation of PTX3 in CSCs may not be inconsistent to its weak expression in bulk tumors. These data might also indicate the possibility that PTX3 may play different roles in CSCs and non-CSCs for tumor regulation. In addition, our data showed that PTX3 expression results in activation of Hedgehog signaling and suppression of Hippo signaling, which might shed light on the unsolved question regarding the functional mechanism of PTX3 in cancer regulation. Nevertheless, the downstream molecular event of PTX3, especially the possible receptor of PTX3 to transmit the signal of the secretory protein into cells, is yet to be discovered before PTX3 targeting becomes practical for cancer treatment.

\section{Methods}

Plasmids and reagents. SH3RF3 cDNA was cloned into pRVPTO-puro-HA ${ }^{64}$, pLVX-puro-Flag, and pLVX-blasticidin (Clonetech), respectively, for overexpression and Co-IP assays. PTX3, JNK1, JNK2 (fused with GFP), JIP1, JIP2, and JIP3 were cloned into PLVX-puro-HA, and MKK4/7 were cloned into pLVX-puroGST for overexpression and Co-IP assays. For SH3RF3 and PTX3 knockdown, shRNAs were designed with the online software sfold ${ }^{65}$, followed by annealing and cloning of the oligonucleotides into pSUPER-retro-puro (Oligo Engine) or pSUPER-retro-blasticidin, with the following target sequences: 5'-ATTTCGAGA TGAAGGACAAAG-3' (SH3RF3 KD1); 5'-TCGAGGAAGGGCCACTATAAT-3' (SH3RF3 KD2); 5'-GGCCGAGAACTCGGATGATTA-3' (PTX3 KD1); 5'-GCC ATGGTGCTTTCAGTTTAA-3' (PTX3 KD2). Sequences for siRNA knockdown, including SH3RF3 siRNA1 (5'-CCAAGAAACGCCACUCCUUTT-3), siRNA2 (5'- 
a

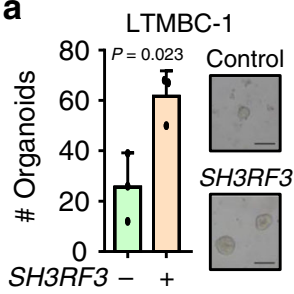

d

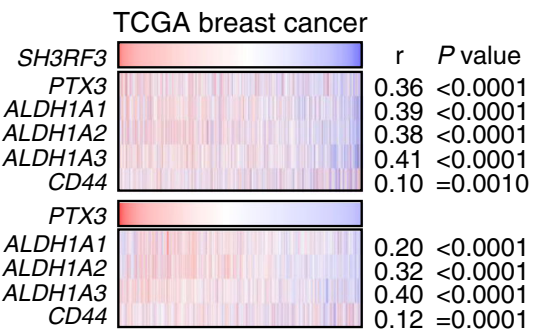

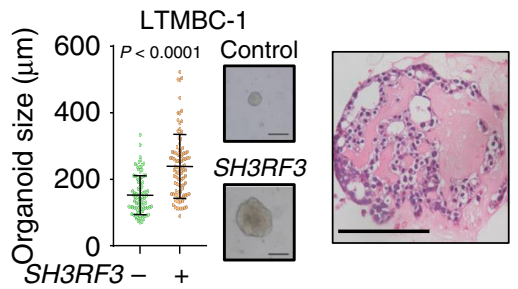

b

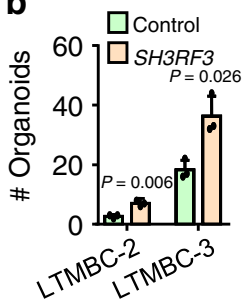

c

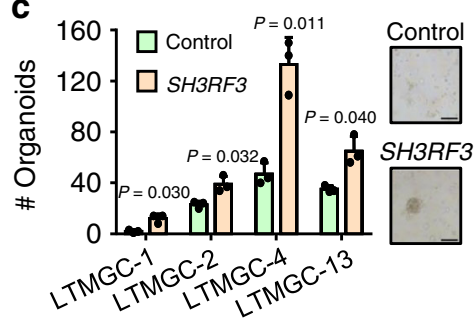

e

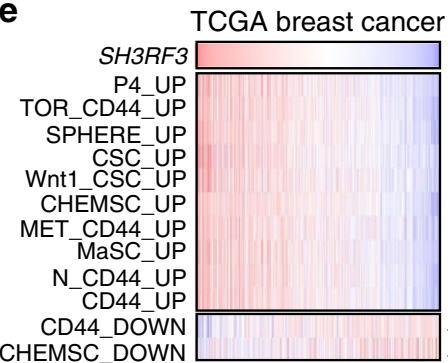

$r \quad P$ value $0.69<0.0001$ $0.72<0.0001$ $0.71<0.0001$ $0.63<0.0001$ $0.61<0.0001$ $0.71<0.0001$ $0.67<0.0001$ $0.68<0.0001$ $0.67<0.0001$ $0.70<0.0001$ $-0.35<0.0001$ $-0.31<0.0001$ $\mathbf{f}$
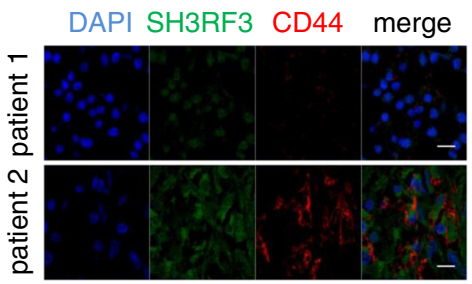

j g

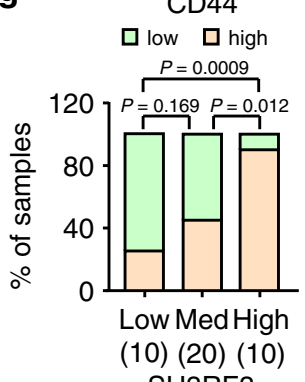

h

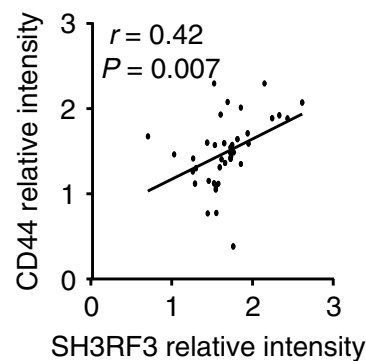

i

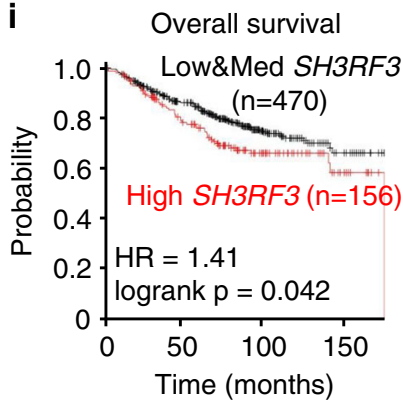

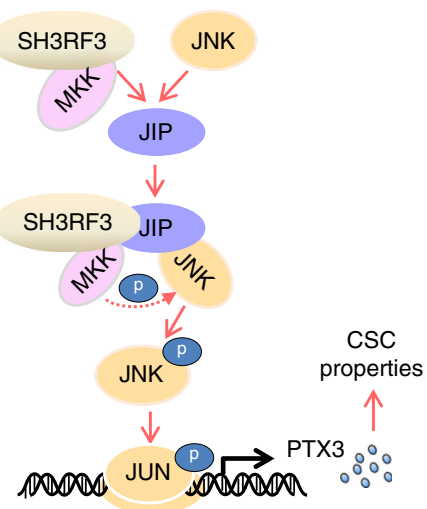

Fig. 8 The clinical relevance of SH3RF3 in human breast tumors. a Patient-derived organoid formation ( $n=3$ organoid culture replicates) in a human breast tumor, LTMBC-1, after SH3RF3 overexpression. (Right panel) Lines represent median values and whiskers indicate the standard deviation. Haematoxylin \& eosin staining of a representative organoid was shown on the right. Scale bar, $100 \mu \mathrm{m}$. $\mathbf{b}$ Patient-derived organoid formation ( $n=3$ organoid culture replicates) of two other human breast tumors (LTMBC-2 and 3) after SH3RF3 overexpression. $\mathbf{c}$ Patient-derived organoid formation ( $n=3$ organoid culture replicates) of four human gastric cancers (LTMGC-1, 2, 4, and 13) with SH3RF3 overexpression. Scale bar, $100 \mu \mathrm{m}$. d Correlation of SH3RF3 and PTX3 expression to different CSC marker genes in TCGA breast cancer cohort ( $n=1097$ patients). e Correlation of SH3RF3 expression and the ssGSEA scores of CSC and MaSC-related gene sets in TCGA breast cancer cohort ( $n=1097$ patients). $\mathbf{f}$ Representative IF images of SH3RF3 and CD44 in breast cancer tissues of the Qilu cohort. Scale bar, $100 \mu \mathrm{m}$. $\mathbf{g}$ CD44 expression levels in breast cancer tissues of the Qilu cohort with different SH3RF3 expression ( $n=40$ patients). Numbers in parenthesis indicate sample sizes. $\mathbf{h}$ Correlation of SH3RF3 and CD44 IF intensities in breast cancer tissues of the Qilu cohort ( $n=40$ patients). i Survival analysis by SH3RF3 expression in Kaplan-Meier Plotter breast cancer cohort $(n=626$ patients). $\mathbf{j}$ Schematic model of the role of SH3RF3 in BCSC regulation. Data represent mean \pm SD. Statistical significance was determined by two-tailed unpaired $t$-test $(\mathbf{a}, \mathbf{b}$ and $\mathbf{c}$ ), chisquared test $(\mathbf{g})$, paired $t$-test $(\mathbf{h})$ or two-sided log-rank test (i). Source data are provided as a Source Data file.

CCCAACGUCAGUGCCGCAATT-3), PTX3 siRNA1 (5'-GCACAAAGAGGA AUCCAUATT-3), siRNA2 (5'-GGGAUAGUGUUCUUAGCAATT-3), JIP3 siRNA1 (5'-GCGGCACACAGAGAUGAUATT-3), siRNA2 (5'-CCAGCACCA CAGGCACCAATT-3), and the scrambled control (5'-UUCUCCGAACGUGU CACGUTT-3) were purchased from GenePharma.

For PTX 3 promoter activity analysis, the -2263 to +52 DNA fragment of the PTX3 gene locus, as well as the truncated and mutated sequences, was cloned into pGL3-basic (Promega) with MluI and BglII sites. All primers used in this study are provided in Supplementary Table 3. All the constructs were verified by DNA sequencing. All cell lines used in this study were tested as mycoplasma free.

For Western blotting, flow cytometry, Co-IP and IF analyses, the following antibodies were used: rabbit anti-human SH3RF3 (ThermoFisher, PA560841), rabbit anti-PTX3 (Proteintech, 13797-1-AP), rabbit anti-human JIP3 (Proteintech, 25212-1-AP), rabbit anti-human JNK2 (Proteintech, 51153-1-AP), rabbit antihuman phospho-JUN (Ser73) (Cell Signaling Technology, 9164S), rabbit antihuman JUN (Cell Signaling Technology, 9165P), rabbit anti-human phosphoSAPK/JNK (Thr183/Tyr185) (Cell Signaling Technology, 4668t), APC mouse antihuman CD24 (Biolegend, 311118), FITC mouse anti-human CD44 (BD

Pharmingen, 555478), PE mouse anti-human CD44 (BD Pharmingen, 555479), Alexa Fluor 488 donkey anti-rabbit IgG (Invitrogen, A-21206), Alexa Fluor 647 goat anti-rat IgG (Biolegend, 405416), rabbit anti-human GAPDH (Sigma, G9545), mouse anti-human $\alpha$-TUBULIN (Sigma, T6199), mouse anti-human $\beta$-ACTIN (Sigma, A2228), rat anti-human CD44 (Santa Cruz, sc-18849), mouse anti-HA (Sigma, H9658), mouse anti-Flag (Sigma, F1804), rabbit anti-HA (Cell Signaling Technology, 3724S). All antibodies were diluted according to the dilution rate recommended in the manufacturers' instructions before use. The inhibitors of various signaling pathways used in this study were BKM120 (MCE, HY-70063), SP600125 (Merck, 420119), BAY11-7082 (Selleck, s2913), and XAV939 (Sigma, $\mathrm{X} 3004)$.

RNA sequencing. Total RNA was extracted from the cells using the Trizol reagent (Invitrogen), and evaluated with a 2100 Bioanalyzer (Aglient Technologies). RNA samples with an RNA integrity number $(\mathrm{RIN})>8$ were used for subsequent sequencing. Library construction and sequencing were performed at WuXi NextCODE, Shanghai. mRNA was enriched from total RNA samples by rRNA removal (HMLER CD44L, CD44H, CD44LS subline cells) or PolyA purification (SH3RF3overexpressing MCF10AT and PTX3-overexpressing HMLER cells), and then fragmented, followed by reverse transcription with random primers. Then the synthesized CDNA was subjected to end-repair, phosphorylation and 'A' base addition according to Illumina's library construction protocol. After Illumina sequencing adapter addition, PCR amplification and purification with AMPure XP Beads (Beckmen), sequencing was performed with a HiSeq X10 sequencing 
platform (Illumina) following Illumina-provided protocols, resulted in $2 \times 150 \mathrm{bp}$ paired-end reads. Skewer v0.2.2 ${ }^{66}$ was used to remove the adapter sequences. Quality control (QC) was performed to remove bases with base quality $<\mathrm{Q} 30$ and reads with length $<=75 \mathrm{bp}$ from subsequent analysis. Data yields of the analyzed samples in this study were 53.90-75.51 M raw reads and 53.81-75.28 M QC-passed reads. Then the filtered reads were mapped to the human genome (hg19) and transcriptome (gencode v19) using the software STAR v2.5.1b ${ }^{67}$ with default parameters ("-outFilterMultimapNmax 10, -outFilterMismatchNmax 10, -outFilterMismatchNoverLmax 0.3, -sjdbOverhang 100, -outFilterType Normal -alignSJoverhangMin 5, -alignSJDBoverhangMin 3, -alignIntronMin 21, -alignIntronMax 0, -alignMatesGapMax 0"), yielding 93.7-97.3\% alignment of the reads. PCR duplication rates of the RNA-sequencing data in this study, estimated by dupRadar $^{68}$, were $0.303 \pm 0.118$ for all the samples. The read duplicates were retained in the following expression analysis in that it was hard to distinguish PCR duplicates from nature duplicates in this sequencing protocol. Expression estimation was performed using RSEM 1.2.2969 with default parameters except the setting "--estimate-rspd". Across-sample normalization and differential expression analysis were performed using the R package EdgeR v3.8.5 ${ }^{70}$ with the trimmed mean of M-values algorithm. Possible multi-mapping of SH3RF3 reads to the whole genome was also analyzed, and it was found that there were $0.6 \%$ SH3RF3 reads multi-mapped to other genes, but none to the other two SH3RF family members, SH3RF1 and SH3RF2. Genes with CPM (count per million) $\geq 5$ (HMLER CD44L, $\mathrm{CD} 44 \mathrm{H}, \mathrm{CD} 44 \mathrm{LS}$ subline cells) or CPM $\geq 1$ (SH3RF3-overexpressing MCF10AT and PTX3-overexpressing HMLER cells) in at least one sample were taken into further analysis. Pairwise comparison between sample groups was performed with exact test for differential expression analysis. Up- and downregulated genes with fold changes $>2$ and Benjamini-Hochberg adjusted $p$ values $<0.05$ were identified.

\section{Bioinformatic analyses of public transcriptomic datasets. The GDC TCGA} Breast Cancer RNAseq (HTSeq-FPKM-UQ) expression dataset was obtained from UCSC Xena ${ }^{71}$. The CSC enrichment of these TCGA tumors were analyzed by ssGESA $^{25}$ with the previously identified gene signature differentially expressed in $\mathrm{CD} 44^{+} \mathrm{CD} 24^{-}$tumor cells as compared with $\mathrm{CD} 44^{-} \mathrm{CD} 24^{+}$counterparts (CD44_UP_DOWN, [26]). Correlation of the ssGSEA scores and expression of each gene was assessed by Pearson correlation analysis. The genes were ranked by Pearson's coefficient $(r)$ and the top 100 positively and negatively correlated genes were used for further analysis. The RNA microarray data of patient-derived tumorspheres and parental tumors were downloaded from the GEO database (GSE7515), and the genes with fold changes $>2$ and Student's $t$-test $P<0.05$ were selected for further analysis. The expression dataset of breast cancer cell lines was obtained from Cancer Cell Line Encyclopedia ${ }^{37}$ and analyzed with ssGSEA. The National Cancer Institute CPTAC (Clinical Proteomic Tumor Analysis Consortium) breast cancer proteomics data were downloaded from the cBioPortal database ${ }^{72,73}$ and used to analyze the correlation of PTX3 expression with cancer prognosis. The full list and description of the CSC or MaSC-related gene sets used in GSEA and ssGSEA were provided in Supplementary Data File 2.

Flow cytometry analyses. Cancer cells harvested from culture during the logarithmic growth period were resuspended in PBS. 500,000 cells were incubated with the antibodies in recommended concentrations by the manufacturer's instructions at $4{ }^{\circ} \mathrm{C}$ for $0.5 \mathrm{~h}$, followed by three times of PBS washing. Then cells were filtered with a 70- $\mu \mathrm{m}$ strainer and sorted by a MoFlo Astrios EQ Flow Cytometer (Beckman) or analyzed by a Gallios Analyzer (Beckman). ALDH activity analysis was performed by the ALDEFLUOR Kit (StemCell Technologies) following the manufacturer's protocol. The specific ALDH inhibitor Diethylaminobenzaldehyde (DEAB) was used as negative control in ALDH analyses. Flow cytometry data processing was performed with FlowJov10 (Tree Star, USA). Flow cytometry gating strategy is shown in Supplementary Fig. 7.

Tumorsphere formation. Cells were cultured in serum-free medium with cell linespecific annexing agents (Supplementary Table 2). 5000-10,000 cells were seeded in 6-well ultra-low attachment plates (\#3471, Corning, USA) and cultured for 1 or 2 weeks. The spheres with diameters larger than $50 \mu \mathrm{m}$ were counted. The detailed information of tumorsphere culture conditions, including culture media, seeded cell numbers, and culture time, of different cell lines was provided in Supplementary Table 2 . The culturing condition of CD44L consecutive tumorsphere passages for CD44LS derivation was the same for the initial tumorsphere culture.

Chemoresistance assays. Five thousand tumor cells were seeded in each well of a 96-well plate. Chemotherapy drugs in various concentrations (paclitaxel 0-200 ng/ $\mathrm{mL}$, doxorubicin $0-500 \mathrm{nM}$ ) or the solvent as the negative control were added into the wells. Cells were cultured for $48 \mathrm{~h}$, followed by MTT assays of cell viability. 3(4,5-dimethyl-2-thiazolyl)-2,5-diphenyl tetrazolium bromide (MTT) was prepared in PBS at a concentration of $5 \mathrm{mg} / \mathrm{mL}$ and $\mathrm{pH} 7.4$. Twenty microliters of MTT solution was added in each well. After $4 \mathrm{~h}$, the crystal was dissolved in DMSO and the absorbance was measured at $490 \mathrm{~nm}$.

Promoter activity assay. The PTX3 promoter activity regulated by JUN was measured by luciferase reporter assays. Two hundred microgram control or JUN plasmids, $200 \mu \mathrm{g}$ PTX3 promoter Firefly luciferase reporter plasmid and $100 \mu \mathrm{g}$ Renilla luciferase plasmid were transfected into HeLa cells in each well of 24-well plates. Cells were cultured for another $48 \mathrm{~h}$ and lysates were collected for luciferase activity analysis in firefly $(25 \mathrm{mM}$ glycylglycine, $15 \mathrm{mM}$ potassium phosphate, 15 $\mathrm{mM} \mathrm{MgSO}_{4}, 2 \mathrm{mM} \mathrm{ATP}, 10 \mathrm{mM}$ DTT, and $1 \mathrm{mM}$ D-luciferin, $\mathrm{pH}$ 7.8) and Renilla $(0.5 \mathrm{M} \mathrm{NaCl}, 1 \mathrm{mM}$ EDTA, $0.1 \mathrm{M}$ potassium phosphate, $0.04 \% \mathrm{BSA}$ and $2 \mu \mathrm{M}$ coelenterazine, $\mathrm{pH}$ 7.4) luciferase assay buffer.

Chromatin immunoprecipitation (ChIP). ChIP assays for PTX3 promoter binding by JUN were conducted in HeLa cells. Briefly, HeLa cells were transfected with JUN-overexpressing or control plasmids. Two days later, cells were crosslinked with $1 \%$ formaldehyde and quenched by $125 \mathrm{mM}$ glycine. Cell nuclear lysate was sonicated and incubated with control IgG or anti-HA antibody for immunoprecipitation. The complex was captured and precipitated by agarose beads. Captured genomic DNA was reverse-crosslinked and purified by ethanol precipitation with Dr. GenTLE Precipitation Carrier (Takara). Purified genomic DNA was used for qPCR analysis.

Co-immunoprecipitation (Co-IP). Cells were transfected with overexpression plasmids $48 \mathrm{~h}$ before Co-IP. The cell lysate was incubated with control IgG or immunoprecipitating primary antibodies overnight at $4{ }^{\circ} \mathrm{C}$. Then, another 2-h incubation was conducted with $25 \mu \mathrm{l}$ protein A/G agarose beads (GE). Beads were washed by lysis buffer for five times and heated at $95^{\circ} \mathrm{C}$ for $10 \mathrm{~min}$. Western blotting was performed to detect the interaction between the concerned proteins.

Immunofluorescence (IF) analysis. Fixed tumor tissues were embedded in paraffin and sectioned into $10-\mu \mathrm{m}$ slices. The sections were dewaxed with xylene and hydrated by a series of ethanol solutions of different concentrations, followed by antigen retrieval. The sections were incubated with SH3RF3 or CD44 primary antibodies overnight at $4{ }^{\circ} \mathrm{C}$, followed by washing with PBS for three times and an incubation with fluorescent-labeled secondary antibodies and DAPI. After washing and sealing, the sections were analyzed by confocal microscopy (ZEISS) and ZEN blue edition software (ZEISS).

Animal studies. Female NSG mice aged at $4-6$ weeks were purchased from Shanghai Model Organisms Center, Inc, maintained in the standard SPF animal house and used in all studies. For tumor transplantation, cancer cells harvested from cell culture were resuspended in PBS at a concentration of $1 \times 10^{7}$ cells $/ \mathrm{mL}$ and diluted to specific concentrations. An incision was made in the abdomen, and the skin was recessed to locate the \#4 mammary fat pad, into which cells were injected under a dissection microscope. Tumor incidence of the mice was determined by palpation and confirmed by surgically opening the mammary glands for tumor examination at the end point of experiments. The tumor was removed from the mice before the volumes were measured. All animal studies were conducted in accordance with Guidelines for the Care and Use of Laboratory Animals that is approved by the Institutional Biomedical Research Ethics Committee of Shanghai Institutes for Biological Sciences.

Clinical samples and patient-derived organoid (PDO) culture. Fresh and paraffin-embedded breast cancer tissues were obtained from Qilu Hospital of Shandong University (Ji'nan, China) with informed patient consent and approval from the Institutional Review Board. Fresh tumor tissues were harvested and transplanted into cleared mammary fat pads of NOD-SCID mice pretreated with CD122 neutralizing antibodies to establish patient-derived xenograft of breast cancers. Tumor cells were dissociated from the established xenografts within two passages and were infected with control or SH3RF3-overexpressing retroviruses. The infected cells were seeded in matrigel in 24-well plates and covered by organoid medium supplemented with 1:50 B27, $0.5 \mu \mathrm{g} / \mathrm{mL}$ Hydrocortisone, $20 \mathrm{ng} / \mathrm{mL}$ bFGF, $20 \mathrm{ng} / \mathrm{mL}$ EGF, $5 \mu \mathrm{g} / \mathrm{mL}$ insulin, and $2 \% \mathrm{FBS}$. After one week of culture, the numbers of organoids were counted.

Statistical analyses. Data analysis was performed using GraphPad Prism 7.0 (GraphPad Software, La Jolla, USA). Unless stated otherwise, two-tailed Student's $t$-test without assumption of equal variance was conducted to compare the data of different groups of in vitro and animal assays. Breast cancer patient survival was analyzed by log-rank test. Pearson correlation coefficient was used to assess the correlation between different data groups. The $p$ values $<0.05$ were regarded as statistically significant. All imaging experiments were repeated at least three times, with similar results.

Reporting summary. Further information on research design is available in the Nature Research Reporting Summary linked to this article.

\section{Data availability}

The RNA sequencing data of SH3RF3 and PTX3-overexpressing cells were deposited in the NODE database (https://www.biosino.org/node) with project IDs OEP000303, OEP000304, and OEP000305 and GEO database (https://www.ncbi.nlm.nih.gov/gds/) 
with project ID GSE130577. The source data underlying Figs. 1b, c, e, f; 2a-c, e; 3a-c, e; 4a-e, g, k; 5a, c, d, f, g; 6; 7; 8a-c, g, h; Supplementary Figs. 1b-e; 2a, b; 3a, c; 4b-d and 5 are provided as a Source Data file. All the other data supporting the findings of this study are available within the article and its Supplementary information files and also from the corresponding author upon reasonable request. A reporting summary for this article is available as a Supplementary Information file.

Received: 3 May 2019; Accepted: 8 April 2020;

Published online: 19 May 2020

\section{References}

1. Burrell, R. A., McGranahan, N., Bartek, J. \& Swanton, C. The causes and consequences of genetic heterogeneity in cancer evolution. Nature 501, 338-345 (2013).

2. Marusyk, A., Almendro, V. \& Polyak, K. Intra-tumour heterogeneity: a looking glass for cancer? Nat. Rev. Cancer 12, 323-334 (2012).

3. Kreso, A. \& Dick, J. E. Evolution of the cancer stem cell model. Cell Stem Cell 14, 275-291 (2014)

4. Gupta, P. B. et al. Stochastic state transitions give rise to phenotypic equilibrium in populations of cancer cells. Cell 146, 633-644 (2011).

5. Al-Hajj, M., Wicha, M. S., Benito-Hernandez, A., Morrison, S. J. \& Clarke, M. F. Prospective identification of tumorigenic breast cancer cells. Proc. Natl Acad. Sci. USA 100, 3983-3988 (2003).

6. Ginestier, C. et al. ALDH1 is a marker of normal and malignant human mammary stem cells and a predictor of poor clinical outcome. Cell Stem Cell $\mathbf{1}$, 555-567 (2007).

7. $\mathrm{Ni}, \mathrm{T}$. et al. Snail1-dependent p53 repression regulates expansion and activity of tumour-initiating cells in breast cancer. Nat. Cell Biol. 18, 1221-1232 (2016).

8. Plaks, V., Kong, N. W. \& Werb, Z. The cancer stem cell niche: how essential is the niche in regulating stemness of tumor cells? Cell Stem Cell 16, 225-238 (2015).

9. Karkkainen, S. et al. Identification of preferred protein interactions by phagedisplay of the human Src homology-3 proteome. EMBO Rep. 7, 186-191 (2006).

10. Karkkainen, S., van der Linden, M. \& Renkema, G. H. POSH2 is a RING finger E3 ligase with Rac1 binding activity through a partial CRIB domain. FEBS Lett. 584, 3867-3872 (2010).

11. Wang, J. et al. A six gene expression signature defines aggressive subtypes and predicts outcome in childhood and adult acute lymphoblastic leukemia. Oncotarget 6, 16527-16542 (2015).

12. Jia, P. et al. Genome-wide association study of HIV-associated neurocognitive disorder (HAND): a CHARTER group study. Am. J. Med. Genet. B Neuropsychiatr. Genet. 174, 413-426 (2017).

13. Lee, J. H. et al. Genetic modifiers of age at onset in carriers of the G206A mutation in PSEN1 with familial Alzheimer disease among Caribbean Hispanics. JAMA Neurol. 72, 1043-1051 (2015).

14. Garlanda, C., Bottazzi, B., Bastone, A. \& Mantovani, A. Pentraxins at the crossroads between innate immunity, inflammation, matrix deposition, and female fertility. Аnпu Rev. Immunol. 23, 337-366 (2005).

15. Salustri, A. et al. PTX3 plays a key role in the organization of the cumulus oophorus extracellular matrix and in in vivo fertilization. Development 131 1577-1586 (2004).

16. Stallone, G. et al. Pentraxin 3: a novel biomarker for predicting progression from prostatic inflammation to prostate cancer. Cancer Res. 74, 4230-4238 (2014).

17. Diamandis, E. P., Goodglick, L., Planque, C. \& Thornquist, M. D. Pentraxin-3 is a novel biomarker of lung carcinoma. Clin. Cancer Res. 17, 2395-2399 (2011).

18. Bonavita, E. et al. PTX3 is an extrinsic oncosuppressor regulating complement-dependent inflammation in cancer. Cell 160, 700-714 (2015).

19. Tsuji, S. et al. Network-based analysis for identification of candidate genes for colorectal cancer progression. Biochem Biophys. Res. Commun. 476, 534-540 (2016).

20. Carmo, R. F. et al. Genetic variation in PTX3 and plasma levels associated with hepatocellular carcinoma in patients with HCV. J. Viral Hepat. 23, 116-122 (2016).

21. Guo, T. et al. The clinical value of inflammatory biomarkers in coronary artery disease: PTX3 as a new inflammatory marker. Exp. Gerontol. 97, 64-67 (2017).

22. Giacomini, A., Ghedini, G. C., Presta, M. \& Ronca, R. Long pentraxin 3: a novel multifaceted player in cancer. Biochim Biophys. Acta Rev. Cancer 1869, 53-63 (2018)

23. Gupta, P. B. et al. Identification of selective inhibitors of cancer stem cells by high-throughput screening. Cell 138, 645-659 (2009).
24. Liu, S. et al. Breast cancer stem cells transition between epithelial and mesenchymal states reflective of their normal counterparts. Stem Cell Rep. 2, 78-91 (2014)

25. Barbie, D. A. et al. Systematic RNA interference reveals that oncogenic KRASdriven cancers require TBK1. Nature 462, 108-112 (2009).

26. Shipitsin, M. et al. Molecular definition of breast tumor heterogeneity. Cancer Cell 11, 259-273 (2007).

27. Creighton, C. J. et al. Residual breast cancers after conventional therapy display mesenchymal as well as tumor-initiating features. Proc. Natl Acad. Sci. USA 106, 13820-13825 (2009).

28. Liu, S. et al. Hedgehog signaling and Bmi-1 regulate self-renewal of normal and malignant human mammary stem cells. Cancer Res. 66, 6063-6071 (2006).

29. Cheon, D. J. et al. A collagen-remodeling gene signature regulated by TGFbeta signaling is associated with metastasis and poor survival in serous ovarian cancer. Clin. Cancer Res. 20, 711-723 (2014).

30. Posokhova, E. et al. GPR124 functions as a WNT7-specific coactivator of canonical beta-catenin signaling. Cell Rep. 10, 123-130 (2015).

31. Yin, J. et al. Pigment epithelium-derived factor (PEDF) expression induced by EGFRvIII promotes self-renewal and tumor progression of glioma stem cells. PLoS Biol. 13 (2015).

32. Bhoopathi, P. et al. MMP-2 mediates mesenchymal stem cell tropism towards medulloblastoma tumors. Gene Ther. 18, 692-701 (2011).

33. Zhang, M. et al. CD133 affects the invasive ability of HCT116 cells by regulating TIMP-2. Am. J. Pathol. 182, 565-576 (2013).

34. Tanimizu, N., Kobayashi, S., Ichinohe, N. \& Mitaka, T. Downregulation of miR122 by grainyhead-like 2 restricts the hepatocytic differentiation potential of adult liver progenitor cells. Development 141, 4448-4456 (2014).

35. Hu, J., Li, G., Zhang, P., Zhuang, X. \& Hu, G. A CD44v+ subpopulation of breast cancer stem-like cells with enhanced lung metastasis capacity. Cell Death Dis. 8, e2679 (2017).

36. Mani, S. A. et al. The epithelial-mesenchymal transition generates cells with properties of stem cells. Cell 133, 704-715 (2008).

37. Marum, L. Cancer Cell Line Encyclopedia launched by Novartis and Broad Institute. Future Med Chem. 4, 947 (2012).

38. Liu, R. et al. The prognostic role of a gene signature from tumorigenic breastcancer cells. N. Engl. J. Med 356, 217-226 (2007).

39. Asselin-Labat, M. L. et al. Control of mammary stem cell function by steroid hormone signalling. Nature 465, 798-802 (2010).

40. Zhao, Q. et al. Identification of genes expressed with temporal-spatial restriction to developing cerebellar neuron precursors by a functional genomic approach. Proc. Natl Acad. Sci. USA 99, 5704-5709 (2002).

41. Yoon, J. W. et al. Gene expression profiling leads to identification of GLI1binding elements in target genes and a role for multiple downstream pathways in GLI1-induced cell transformation. J. Biol. Chem. 277, 5548-5555 (2002).

42. Cordenonsi, M. et al. The Hippo transducer TAZ confers cancer stem cellrelated traits on breast cancer cells. Cell 147, 759-772 (2011).

43. Takebe, N. et al. Targeting Notch, Hedgehog, and Wnt pathways in cancer stem cells: clinical update. Nat. Rev. Clin. Oncol. 12, 445-464 (2015).

44. Mo, J. S., Park, H. W. \& Guan, K. L. The Hippo signaling pathway in stem cell biology and cancer. EMBO Rep. 15, 642-656 (2014)

45. Thomas, C. et al. Pentraxin-3 is a PI3K signaling target that promotes stem cell-like traits in basal-like breast cancers. Sci. Signal. 10, eaah4674 (2017).

46. Ozanne, B. W., Spence, H. J., McGarry, L. C. \& Hennigan, R. F. Transcription factors control invasion: AP-1 the first among equals. Oncogene 26, 1-10 (2007).

47. Matthews, C. P. et al. Dominant-negative activator protein 1 (TAM67) targets cyclooxygenase- 2 and osteopontin under conditions in which it specifically inhibits tumorigenesis. Cancer Res. 67, 2430-2438 (2007).

48. Yasuda, J., Whitmarsh, A. J., Cavanagh, J., Sharma, M. \& Davis, R. J. The JIP group of mitogen-activated protein kinase scaffold proteins. Mol. Cell Biol. 19, 7245-7254 (1999).

49. Sachs, N. et al. A living biobank of breast cancer organoids captures disease heterogeneity. Cell 172, 373-386 e310 (2018).

50. Lanczky, A. et al. miRpower: a web-tool to validate survival-associated miRNAs utilizing expression data from 2178 breast cancer patients. Breast Cancer Res. Treat. 160, 439-446 (2016).

51. Zhang, Q. G. et al. Knock-down of POSH expression is neuroprotective through down-regulating activation of the MLK3-MKK4-JNK pathway following cerebral ischaemia in the rat hippocampal CA1 subfield. $J$. Neurochemistry 95, 784-795 (2005).

52. Alroy, I. et al. The trans-Golgi network-associated human ubiquitin-protein ligase POSH is essential for HIV Woe 1 production. Proc. Natl Acad. Sci. USA 102, 1478-1483 (2005).

53. Xu, Z., Kukekov, N. V. \& Greene, L. A. POSH acts as a scaffold for a multiprotein complex that mediates JNK activation in apoptosis. EMBO J. 22, 252-261 (2003). 
54. Wilhelm, M. et al. Sh3rf2/POSHER protein promotes cell survival by ringmediated proteasomal degradation of the c-Jun $\mathrm{N}$-terminal kinase scaffold POSH (Plenty of SH3s) protein. J. Biol. Chem. 287, 2247-2256 (2012).

55. Deng, Q. et al. Imputation-based association analyses identify new lung cancer susceptibility variants in CDK6 and SH3RF1 and their interactions with smoking in Chinese populations. Carcinogenesis 34, 2010-2016 (2013).

56. Chaffer, C. L. et al. Normal and neoplastic nonstem cells can spontaneously convert to a stem-like state. Proc. Natl Acad. Sci. USA 108, 7950-7955 (2011).

57. Fang, M. et al. IL33 promotes colon cancer cell stemness via JNK activation and macrophage recruitment. Cancer Res. 77, 2735-2745 (2017).

58. Yoon, C. H. et al. c-Jun N-terminal kinase has a pivotal role in the maintenance of self-renewal and tumorigenicity in glioma stem-like cells. Oncogene 31, 4655-4666 (2012).

59. Kitajima, S. et al. The RB-IL-6 axis controls self-renewal and endocrine therapy resistance by fine-tuning mitochondrial activity. Oncogene 36, 5145-5157 (2017).

60. Scimeca, M. et al. Emerging prognostic markers related to mesenchymal characteristics of poorly differentiated breast cancers. Tumour Biol. 37, 5427-5435 (2016).

61. Chi, J. Y. et al. Targeting chemotherapy-induced PTX3 in tumor stroma to prevent the progression of drug-resistant cancers. Oncotarget 6, 23987-24001 (2015).

62. Ronca, R. et al. Long pentraxin-3 inhibits epithelial-mesenchymal transition in melanoma cells. Mol. Cancer Ther. 12, 2760-2771 (2013).

63. Ronca, R. et al. Long-pentraxin 3 derivative as a small-molecule FGF trap for cancer therapy. Cancer Cell 28, 225-239 (2015).

64. Celia-Terrassa, T. et al. Normal and cancerous mammary stem cells evade interferon-induced constraint through the miR-199a-LCOR axis. Nat. Cell Biol. 19, 711 (2017).

65. Ding, Y. \& Lawrence, C. E. A statistical sampling algorithm for RNA secondary structure prediction. Nucleic Acids Res. 31, 7280-7301 (2003).

66. Jiang, H., Lei, R., Ding, S. W. \& Zhu, S. Skewer: a fast and accurate adapter trimmer for next-generation sequencing paired-end reads. BMC Bioinforma. 15, 182 (2014)

67. Dobin, A. et al. STAR: ultrafast universal RNA-seq aligner. Bioinformatics 29, 15-21 (2013)

68. Sayols, S., Scherzinger, D. \& Klein, H. dupRadar: a Bioconductor package for the assessment of PCR artifacts in RNA-Seq data. BMC Bioinforma. 17, 428 (2016).

69. Li, B. \& Dewey, C. N. RSEM: accurate transcript quantification from RNA-Seq data with or without a reference genome. BMC Bioinforma. 12, 323 (2011).

70. Robinson, M. D., McCarthy, D. J. \& Smyth, G. K. edgeR: a Bioconductor package for differential expression analysis of digital gene expression data. Bioinformatics 26, 139-140 (2010).

71. Goldman, M. et al. The UCSC Xena platform for public and private cancer genomics data visualization and interpretation. Preprint at https://www. biorxiv.org/content/10.1101/326470v6:326470 (2019).

72. Cerami, E. et al. The cBio cancer genomics portal: an open platform for exploring multidimensional cancer genomics data. Cancer Disco. 2, 401-404 (2012).

73. Gao, J. et al. Integrative analysis of complex cancer genomics and clinical profiles using the cBioPortal. Sci. Signal 6, pl1 (2013).

\section{Acknowledgements}

We thank Yan Ji, Xiang Miao, Kai Wang, Yujia Zhai, Chengji Wang, Yiting Yuan, Fengtao Qian, Shuyang Yan, Tao Huang, Jun Li, and Zhonghui Weng at the Institute of Nutrition and Health core facilities for technical support. The study was funded by National Natural Science Foundation of China (81430070, 81661148048, 81725017, 81802647), Chinese Academy of Sciences (QYZDB-SSW-SMC013), Ministry of Science and Technology of China (2017YFA0103502), the Program of Shanghai Academic Research Leader (19XD1404500), and Natural Science Foundation of Heilongjiang Province of China (QC2017111).

\section{Author contributions}

G.H. was responsible for experimental design, data analysis, and the supervision of this project. P.Z. and Y.L. performed most of experiments and mainly contributed to writing the paper. C.L., X.L., and M.C. performed the PDO assays. X.C. and G.W. contributed to quality control of RNA sequencing. Y.W., P.T., and X.Z. contributed to the immunofluorescence analysis of clinical samples. T.L. contributed to the collection and analysis of clinical samples.

\section{Competing interests}

The authors declare no competing interests.

\section{Additional information}

Supplementary information is available for this paper at https://doi.org/10.1038/s41467020-16051-9.

Correspondence and requests for materials should be addressed to T.L. or G.H.

Peer review information Nature Communications thanks Rumela Chakrabarti, JoseEzequiel Martin and the other, anonymous, reviewer(s) for their contribution to the peer review of this work. Peer reviewer reports are available.

Reprints and permission information is available at http://www.nature.com/reprints

Publisher's note Springer Nature remains neutral with regard to jurisdictional claims in published maps and institutional affiliations.

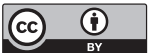

Open Access This article is licensed under a Creative Commons Attribution 4.0 International License, which permits use, sharing, adaptation, distribution and reproduction in any medium or format, as long as you give appropriate credit to the original author(s) and the source, provide a link to the Creative Commons license, and indicate if changes were made. The images or other third party material in this article are included in the article's Creative Commons license, unless indicated otherwise in a credit line to the material. If material is not included in the article's Creative Commons license and your intended use is not permitted by statutory regulation or exceeds the permitted use, you will need to obtain permission directly from the copyright holder. To view a copy of this license, visit http://creativecommons.org/ licenses/by/4.0/.

(C) The Author(s) 2020 